\title{
études expérimentale et théorique du comportement de la grave non traitée
}

\author{
on the behaviour of non treated gravel, \\ experimental and numerical aspects
}

\author{
M. BOUASSIDA \\ École Nationale d'Ingénieurs de Tunis, Département Génie Civil *
}

\section{Résumé}

La grave non traitée est couramment utilisée en construction routière. Pour ce matériau on justifie le choix d'un modèle de comportement du type élastoplastique avec écrouissage isotrope à partir d'observations réalisées à l'appareil triaxial. Lors d'une étude expérimentale sur une grave non traitée $0 / 14$, un bon calage des paramètres du modèle est obtenu. La simulation des essais triaxiaux réalisés montre que ce modèle décrit assez bien le comportement du matériau testé surtout pour les petites déformations. La mise en œuvre numérique du modèle retenu est faite par éléments finis. Le calcul d'une chaussée souple montre que le modèle est plus réaliste que celui de l'élasticité linéaire qui conduit à des résultats incompatibles avec les caractéristiques du matériau. En considérant deux modes de chargement différents (contrainte imposée, déplacement imposé), on montre que le modèle permet d'obtenir une charge maximale pour un massif homogène constitué par la grave. Une étude comparative des deux modes de chargement est entreprise.

\section{Abstract}

Non treated gravel is currently used in road construction. For this material an elastoplastic model, with isotropic hardening, has been adopted following the experimental behaviour observed from experiments. An experimental study on a 0/14 non treated gravel has been carried out. The parameters of the model being derived from the experimental results. The simulation of the tests showed up that the retained model gave a good description especially for small strains. The numerical computations were made by the finite element method. The design of a flexible pavement gave more realistic results than those obtained by the linear elasticity assumption. By considering two load conditions (imposed stress, imposed displacement), the calculation with the model gave a maximum load for an homogeneous half space constituted with the 0/14 non treated gravel. A comparison between the two load conditions was also investigated. 


\section{CONVENTIONS}

On utilise la convention d'Einstein sur les indices muets.

Les contraintes sont comptées positives en compression.

Le produit deux fois contracté des tenseurs a et $b$ est noté a.b.

La dérivée par rapport au temps (ou l'incrément infinitésimal) de la grandeur a est notée à.

$\left(\mathrm{a}^{+}=\right.$a si a $>0 ;(\mathrm{a})^{+}=0$ si non.

\section{PRINCIPALES NOTATIONS}

'a : la transposée de la matrice représentative du tenseur a.

$\sigma$ : tenseur des contraintes; $\sigma_{1}, \sigma_{2}, \sigma_{3}$ : valeurs principales.

s : déviateur de $\sigma$.

$\mathcal{E}^{(p)}$ : tenseur des déformations (plastiques); $\varepsilon_{1}^{(p)}, \varepsilon_{2}^{(p)}$, $\varepsilon_{3}^{(p)}$ : valeurs principales.

$\mathrm{m}$ : déviateur de $\varepsilon^{p}$

$l_{1}=\sigma_{i j} ; \quad J_{2}=\left(s_{i j} \cdot s_{i j}\right) / 2$

$\mathrm{e}_{1}^{(\mathrm{p})}=\varepsilon_{11}^{(p)} ; \mathrm{N}=\left(\mathrm{m}_{\mathrm{ij}}, \mathrm{m}_{\mathrm{l}}\right) / 2$.

$\mathrm{E}$ : tenseur d'élasticité d'ordre 4.

$\mathbb{E}$ : module d'élasticité ; v : coefficient de Poisson.

$k=(1-2 v) / E$ : module de compressibilité.

$\mathrm{C}$ : cohésion; $\varphi$ : angle de frottement.

$\mathrm{n}$ : normale sortante au domaine $(\Omega)$.

Essai triaxial

$\sigma_{1}, \sigma_{3}$ sont les contraintes principales maximale et minimale.

$\mathrm{q}=\sigma_{1}-\sigma_{3}$ : contrainte déviatorique.

$\mathrm{p}=\left(\sigma_{1}+2 \sigma_{3}\right) / 3$ : contrainte moyenne.

\section{INTRODUCTION}

La plasticité appliquée aux sols date de très longtemps avec, en premier lieu, le critère de rupture de COU. LOMB (1773). Ensuite, d'autres modèles ont été proposés, ils admettent un domaine d'élasticité non fermé dans l'espace des contraintes (par exemple DRUCKER et al., 1951). Cette hypothèse, qui est discutable, a été éliminée avec l'apparition de modélisations à domaines d'élasticité fermés; la première est celle proposée par DRUCKER et al. (1957). Les modèles qui l'ont suivie considèrent les résultats d'essais à trajets de charge imposés (en particulier l'essai triaxial).

Pour mieux simuler le comportement des sols, ces modèles, de plus en plus nombreux, se compliquent davantage avec l'introduction de nouvelles hypothèses (plasticité non associée, écrouissage cinématique, etc.); (MROZ et al., 1982). Cependant l'usage de ces modèles reste réduit pour des applications réelles en génie civil. Généralement on se limite à simuler les résultats expérimentaux par des calculs analytiques ou numériques obtenus par le modèle pour justifier son choix. Parmi les modèles ayant fait l'objet de calcul d'ouvrages, on cite ceux du CAM-CLAY qui ont été appliqués pour les remblais de KINGS LYNN (1972).
Le but de ce travail est l'étude du comportement de la grave, en vue de l'appliquer au calcul de structures constituées par ce matériau.

Le modèle élastique linéaire appliqué au calcul des structures de chaussées souples à base de ce matériau conduit à des résultats aberrants (contraintes de traction inadmissibles pour la grave) (FRIÂA et al., 1983)

Pour établir un dimensionnement plus rationnel des chaussées souples, on utilise un modèle élastoplastique avec écrouissage isotrope pour la grave.

On justifie, en premier lieu, le choix du modèle retenu à partir d'une étude expérimentale réalisée à l'appareil triaxial classique sur une grave non traitée $0 / 14$. En second lieu, nous donnons les résultats de calage des paramètres du modèle. On présente ensuite les types de sollicitations retenus pour le calcul des structures (massif en grave, chaussée souple) : chargement avec contrainte imposée et chargement avec déplacement imposé. On écrit la formulation variationnelle du problème en considérant le modèle « déplacement $*$. La mise en ceuvre numérique est faite par la méthode des éléments finis. On donne les principaux résultats de simulation des essais triaxiaux et des calculs de structures. Une étude comparative entre les deux modes de chargement est donnée à la fin.

\section{COMPORTEMENT OBSERVÉ DE LA GRAVE}

La grave a fait l'objet d'études expérimentales réalisées au moyen de l'appareil triaxial classique, sous l'action de sollicitations quasi statique et dynamique [3], [20]. On donne ci-dessous quelques résultats relatifs au comportement observé de ce matériau.

\section{Déformation volumique plastique}

Au cours d'un cycle charge-décharge en consolidation isotrope on observe ce type de déformation (fig. 1); pour un nouveau chargement on constate la présence de points anguleux sur le cycle (fig. 1). Cette déformation n'est pas alors affectée par la viscosité (MANDEL. 1978). En outre, comme elle évolue au cours du chargement, cette déformation constitue un paramètre d'écrouissage, Suivant son signe, on dira que le matériau est contractant $\left(e^{p_{1}}<0\right)$ ou dilatant $\left(e^{p_{1}}>0\right)$.

\section{État caractéristique}

Ce nouveau concept, qui a été formulé sur la base des travaux de KIRKPATRICK (1961), HABIB et LUONG (1978), est observé sur la grave. Lors d'un cisaillement à contrainte latérale constante, le matériau subit une diminution de volume (contractance) jusqu'à une valeur minimum qui correspond à l'état caractéristique, à partir de laquelle le volume augmente (dilatance) jusqu'à l'écoulement (fig. 2).

\section{Variation de volume à l'écoulement (état critique)}

L'état critique, comme il a été formulé par WROTH et al. (1968), n'est pas observé sur la grave. L'écoulement du matériau lors d'un cisaillement n'est pas 
parfait, il a lieu avec variation de volume (fig. 3). La déformation volumique plastique de cisaillement $\sqrt{N}$ constitue donc un paramètre d'écrouissage.

\section{Cohésion - angle de frottement}

La représentation, dans le diagramme de MOHRCOULOMB, des résultats d'essais triaxiaux obtenus sur la grave (fig. 4) montre qu'il s'agit d'un matériau à cohésion non négligeable, avec un angle de frottement élevé.

\section{MODÉLISATION DE LA GRAVE NON TRAITÉE}

Afin de rendre compte des phénomènes expérimentaux, observés, à partir de l'essai triaxial classique, une modélisation adéquate du comportement de la grave peut être conçue à l'aide d'un modèle élastoplastique.

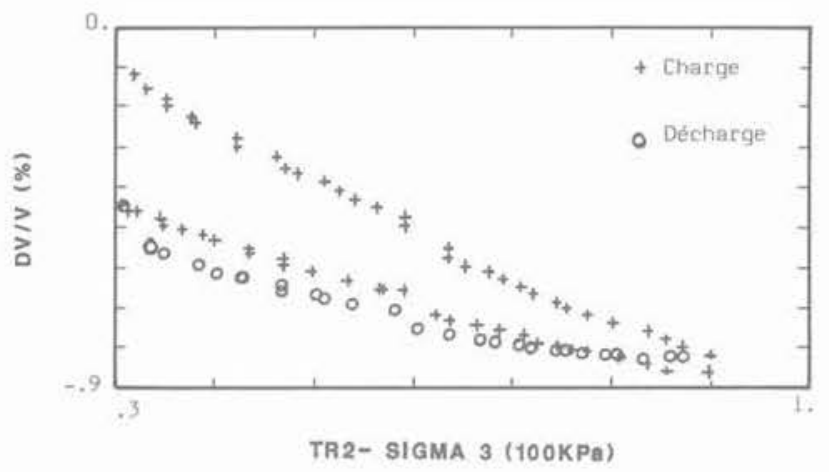

Fig. 1. - Evolution de la déformation volumique dans une consolidation isotrope (charge - décharge - recharge).

Fig. 1. - Variation of volumic strain with stress in isotropic consolidation.

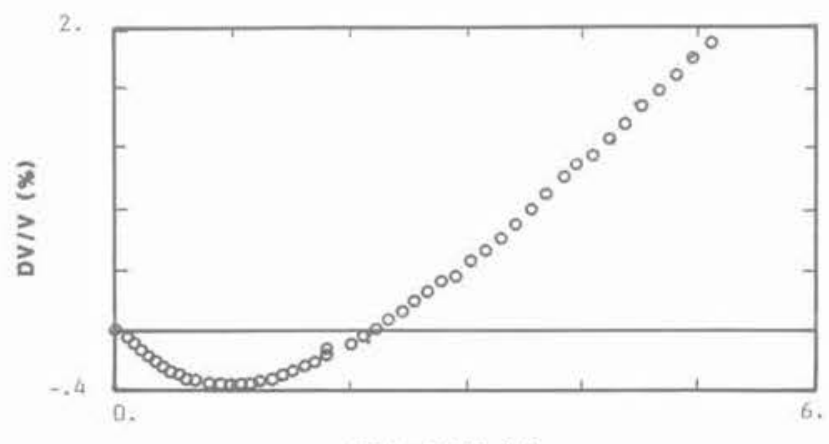

TR1- DH/H (\%)

Fig. 2. - Evvolution de la déformation volumique dans un cisaillement à contrainte latérale constante. Fig. 2. - Variation of volumic strain with axial strain in triaxial shear.
Différents modèles ont été proposés pour décrire le comportement des sols d'une manière générale et en particulier celui des matériaux granulaires. Une présentation des modèles est donnée dans [2] ; nous citons quelques-uns sans chercher à les décrire en détail.

\section{Les modèles Cam-Clay}

Ces modèles, qui découlent de la théorie de l'état critique, ont été développés à l'université de Cambridge par ROSCOE et al. (1963), puis BURLAND (1967); ils sont destinés à décrire le comportement des argiles. La plasticité associée avec l'hypothèse d'écrouissage isotrope constitue le cadre de ces modèles [17], pour lesquels deux versions ont été proposées : le Cam-Clay initial et le Cam-Clay modifié, Ils ont été développés aussi par d'autres auteurs PENDER (1977), PREVOST et al. (1975), etc.

\section{Modèle de LADE et DUNCAN}

Proposé par LADE et al. (1975), ce modèle est placé dans le cadre de la plasticité non associée. Il a été vérifié par des essais triaxiaux cubiques et de torsion-cisaillement sur du sable. Une étude par LADE (1977) a permis d'améliorer le modèle pour tenir compte des déformations plastiques de cisaillement pouvant apparaître dans une consolidation isotrope. Une vérification expérimentale a été faite sur trois matériaux non cohérents. Le modèle simulant bien les résultats expérimentaux obtenus, AUBRY et al. (1979) en font un algorithme par éléments finis.

\section{Modèle de VERMEER}

C'est un modèle à écrouissage double, proposé dans le cadre de la plasticité non associée; en particulier on y fait intervenir l'équation de contrainte-dilatance de ROWE pour prendre en compte le cisaillement. Il a été proposé au début pour les sols pulvérulents sollicités par un chargement initial suivi d'un déchargementrechargement (1978). Puis il a été généralisé pour les matériaux granulaires lâches et cohérents (1984) en faisant intervenir la cohésion et l'angle de frottement.

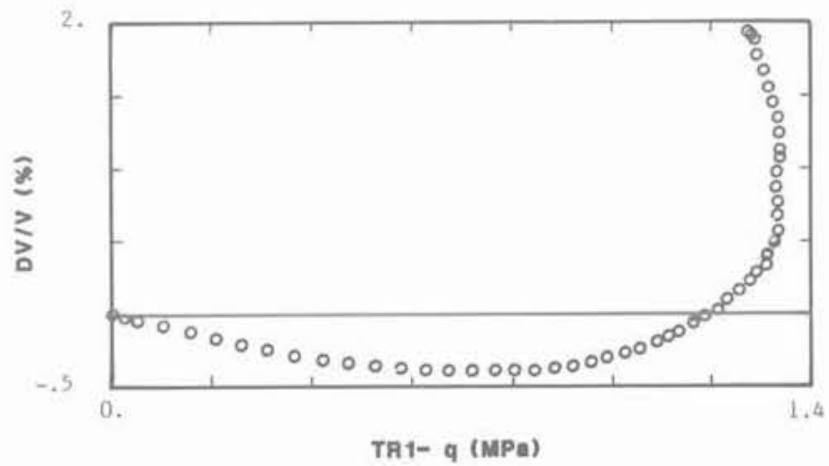

Fig. 3. - Evolution de la contrainte déviatorique en fonction de la variation du volume.

Fig. 3. - Variation of volumic strain with deviatoric stress in triaxial shear. 


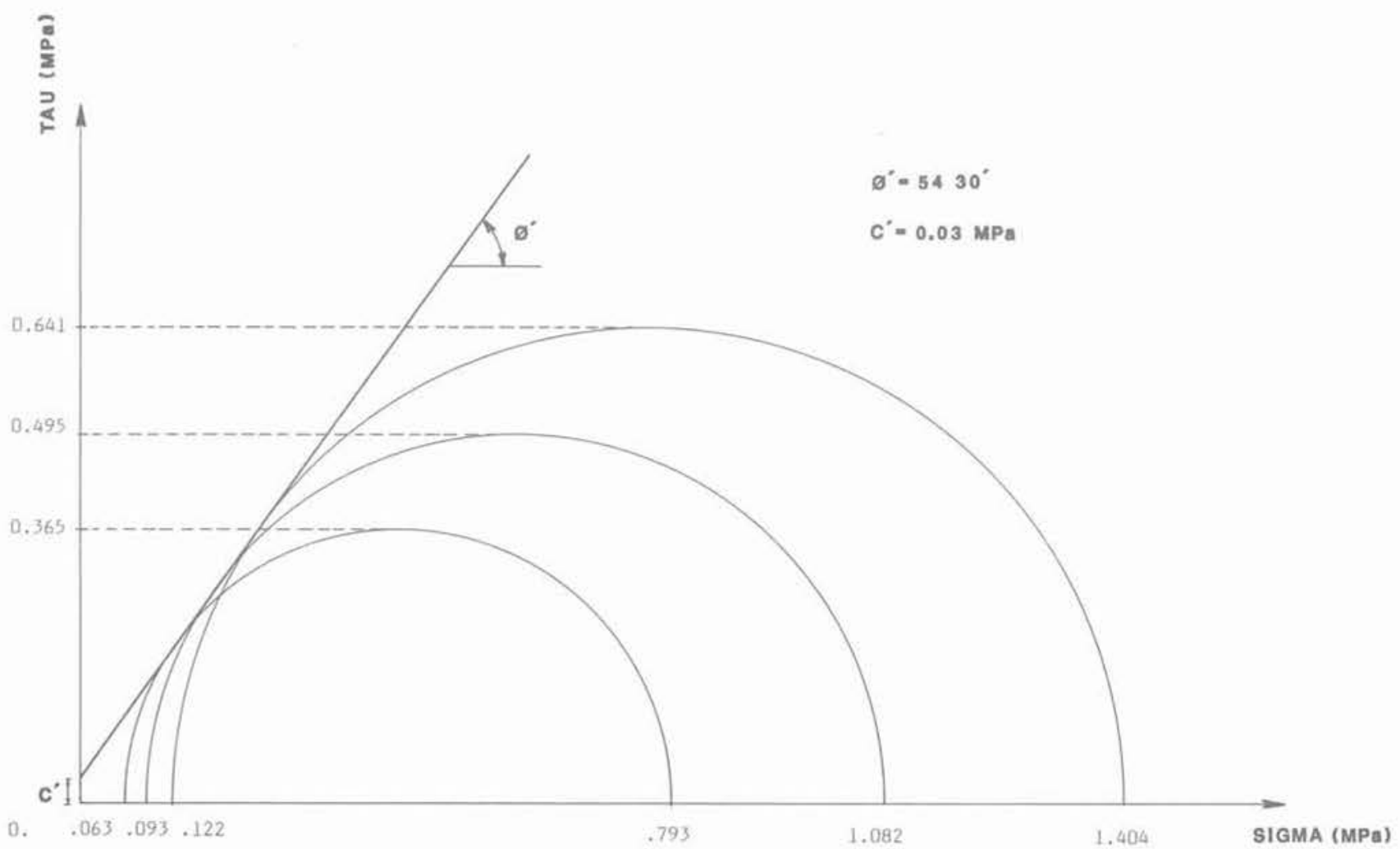

Fig. 4. - Courbe intrinsèque de la G.N.T. 0/14.

Fig. 4. - Failure envelope for drained tests on non treated gravel samples.

\section{Modèles à écrouissage anisotrope}

L'hypothèse d'écrouissage isotrope est souvent considérée dans les modèles élastoplastiques pour raison de simplicité. Afin de rendre compte de l'anisotropie des sols, des modèles à écrouissage anisotrope ont été proposés, on cite PRÉVOST $(1977,1979)$; MROZ et al. (1982), etc.

\section{Modèle retenu pour la grave}

Il est obtenu par la combinaison de deux modèles proposés par WILDE $(1977,1979)$ pour les matériaux granulaires dans le cadre de la plasticité associée avec écrouissage isotrope. Une présentation détaillée de ces modèles est donnée dans [34]. Le critère de plasticité, représenté sur la figure 5, est donné par l'équation (1).

$\mathrm{F}=\sqrt{J_{2}}+\left(\mathrm{MI}_{1}+\mathrm{c}\right)\left(\mathrm{Ln}\left(\mathrm{Ml}_{1}+\mathrm{c}\right) /\left(\mathrm{MI}_{1}{ }^{*}+\mathrm{c}\right)-\right.$ $\left.(K / M)\left(e_{1}^{p}-6\left(M^{*}-M\right)\right) \sqrt{N}\right)$

On fait intervenir cinq paramètres dans ce modèle, qui sont :

$\mathrm{I}_{1}^{*}$ : la trace du tenseur $\sigma$ correspondant à la première surface de charge.

$\mathrm{M}$ : pente de la ligne "critique * : le taux de déformation volumique plastique est nul.

$\mathrm{K}$ : une constante adimensionnelle relative à l'écrouissage.
$\mathrm{M}^{*}$ : pente de la ligne d'écoulement : l'écrouissage en contraintes est nul. c : une constante qui est fonction de la cohésion et l'angle du frottement du matériau, on établit dans [3] que :

$$
c=\left(m_{c} C \operatorname{cotg} \varphi\right) / \sqrt{3}(\cdot)
$$

Dans ce modèle on fait intervenir les invariants $I_{1}$ et $\sqrt{J_{2}}$, qui sont indépendants dans l'essai triaxial classique, et les invariants $e_{1}^{p}$ et $\sqrt{\mathrm{N}}$ (similaires aux précédents) afin de prendre en compte l'écrouissage du matériau en termes des déformations plastiques. L'hypothèse de WILDE (1979) postulant que l'introduction de l'invariant $\sqrt{\mathrm{N}}$ dans ce critère a pour but la prise en compte de la variation de volume à l'écoulement, peut être montrée à partir des équations du modèle. La règle de normalité s'écrit :

$$
\dot{\varepsilon}_{i j}^{p}=\dot{\lambda} \delta \mathrm{F} / \delta \sigma_{i j}
$$

on fait l'hypothèse de la charge plastique qui se traduit par les conditions :

$$
\begin{aligned}
& \mathrm{F}=0 \\
& \dot{\mathrm{F}}=0 \\
& \dot{\lambda}>0
\end{aligned}
$$

(") $\mathrm{m}_{\mathrm{c}}$ est la pente de la ligne critique dans le plan $\mathrm{p}$-q, pour l'essai triaxial en compression; on a : $\mathrm{m}_{\mathrm{c}}=6 \sin \phi /(3-\sin \phi)$. 
l'écriture de la loi incrémentale en élastoplasticité, d'après [3], permet la détermination du multiplicateur de plasticité qui s'écrit :

- en contraintes $: \dot{\lambda}=((' \delta \mathrm{F} / \delta \sigma) . \sigma)) / \mathrm{h}_{a}$

avec :

$$
\mathrm{h}_{\mathrm{s}}=-\left({ }^{1} \delta \mathrm{F} / \delta \sigma\right) \cdot\left(\delta \mathrm{F} / \delta \varepsilon^{\mathrm{p}}\right)
$$

- en déformations: $\quad \dot{\lambda}=\left(((\delta \mathrm{F} / \delta \sigma) . \mathbb{E}, \dot{\varepsilon})+/ \mathrm{h}_{\varepsilon}\right.$

avec : $\quad h_{\varepsilon}=h_{\sigma}+\left({ }^{\prime} \delta \mathrm{F} / \delta \sigma\right)$. $\mathbb{E} \cdot(\delta \mathrm{F} / \delta \sigma)$

$h_{g}$ et $h_{\varepsilon}$ sont les termes d'écrouissage en contraintes et en déformations.

La différentielle totale du critère s'écrit :

$$
\begin{gathered}
\dot{\mathrm{F}}=\left(\delta \mathrm{F} / \delta \sqrt{J_{2}}\right) \sqrt{J_{2}}+\left(\delta \mathrm{F} / \delta I_{1}\right) \dot{\mathrm{I}}_{1} \\
+\left(\delta \mathrm{F} / \delta e_{1}^{p}\right) \dot{e}_{1}^{p}+\left(\delta \mathrm{F} / \delta e_{1}^{p}\right) /(\delta \mathrm{F} / \delta \sqrt{\mathrm{N}})
\end{gathered}
$$

Dans la phase d'écoulement pour le cas du cisaillement triaxial à contrainte latérale constante, le déviateur des contraintes et la trace de $\sigma$ sont constants, d'où :

$$
\begin{gathered}
\sqrt{\dot{J}_{2}}=\dot{q}=0 \\
\dot{I}_{1}=0 .
\end{gathered}
$$

D'après la règle de normalité (2), et tenant compte des conditions (3a), (7a) et (7b), on peut écrire à partir de (6) :

$$
(\delta \mathrm{F}) /(\delta \sqrt{\mathrm{N}})=-3\left(\delta \mathrm{F} / \delta \mathrm{I}_{1}\right) \cdot\left(\delta \mathrm{F} / \delta \mathrm{e}_{1}^{\mathrm{p}}\right) / \cos \mathrm{x}
$$

avec :

$$
\cos x=s_{i j} \cdot m_{i j} /\left(2 \sqrt{J_{2}} \sqrt{N}\right)^{(\cdot)}
$$

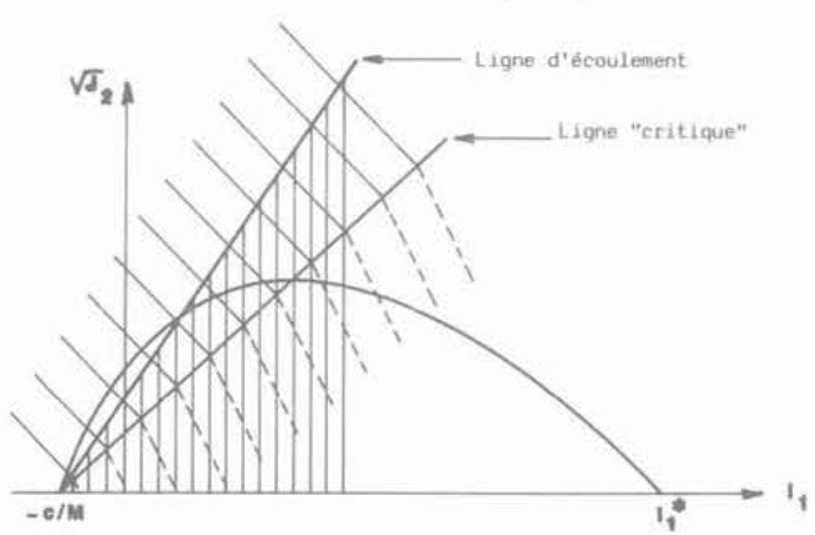

Ecrouissage positif en contrainte
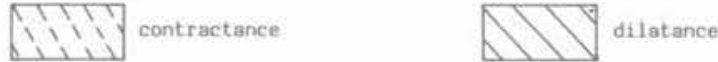

Fig. 5. - Représentation du critère de plasticité pour le modèle; d'après Bouassida et al. (1984).

Fig. 5. - The yield surface for the retained model; from Bouassida and al.

(*) Pour l'essai triaxial classique, on a $\cos x=1$.
A partir de (2) l'équation (8), d'après (9) s'écrit :

$$
\delta \mathrm{F} / \delta \sqrt{\mathrm{N}}=-\dot{e} \dot{p}\left(\delta \mathrm{F} / \delta e_{\mathrm{p}}^{\mathrm{p}}\right) /(\dot{\lambda} \cos \mathrm{x})
$$

les termes $\delta \mathrm{F} / \delta e_{\mathfrak{p}}^{\mathrm{p}}, \dot{\lambda}$, et cos $\mathrm{x}$ sont indépendants de $\dot{e} \mathfrak{p}$ [3] ; à partir de (10) on voit que la dépendance du critère en $\sqrt{\mathrm{N}}$ traduit bien la variation de volume du matériau à l'écoulement.

Sur la figure 5 , où on représente le critère de plasticité, on distingue :

- la zone de contractance : $e_{1}^{p}<0$;

- la zone de dilatance : $e_{1}^{p}>0$;

les deux zones précédentes sont séparées par l'état "critique " (qui est similaire à l'état critique classique) pour lequel le taux de déformation volumique plastique s'annule, mais il n'y a pas d'écoulement (au sens global) du matériau. Pour l'essai triaxial classique on montre que l'état caractéristique, défini par LUONG (1978), a lieu à un niveau de contraintes supérieur à celui de l'état « critique » [3].

La zone d'écrouissage négatif en contraintes correspond à un état instable du matériau. Sur la ligne de pente $\mathrm{M}^{*}$ a lieu l'écoulement (au sens global) du matériau où l'écrouissage en contraintes est nul.

\section{Solutions analytiques obtenus par le modèle}

En considérant des trajets de charge où les contraintes sont imposées dans une direction, on établit des solutions analytiques pour les cas des matériaux cohérent et non cohérent [3]. Sur la figure 6, on voit l'influence de la cohésion qui.fait diminuer la déformation plastique.

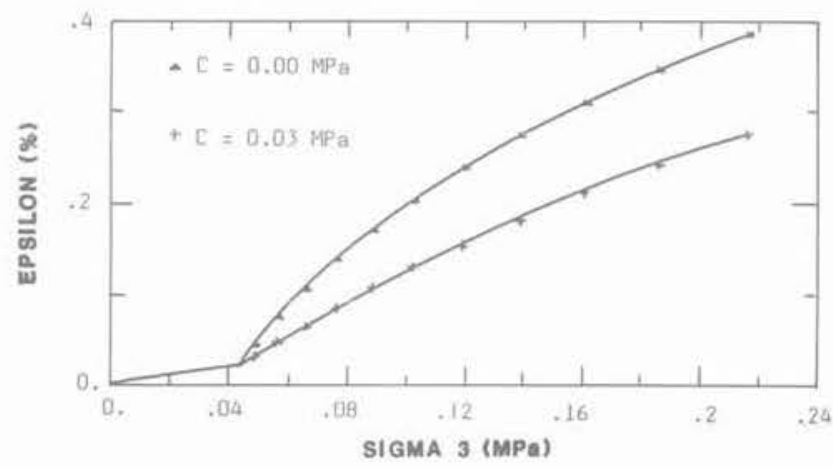

Fig. 6. - Influence de la cohésion sur l'évolution de la déformation dans une consolidation isotrope. Fig. 6. - Cohesion influence on strain evolutian in isotropic consolidation.

Le modèle ainsi construit, rend compte qualitativement des phénomènes expérimentaux observés sur la grave. En outre, on y fait intervenir un petit nombre de paramètres, et leur détermination expérimentale est facile. Elle se fait à partir d'un essai triaxial classique, dans lequel on réalise une charge-décharge-recharge en consolidation isotrope suivie par un cisaillement à 
contrainte latérale constante. La méthode de détermination des paramètres, décrite dans [3], peut être résumée comme suit :

- en consolidation isotrope on détermine le module de compressibilité $\mathrm{k}$ dans la décharge. En charge, on déduit la déformation volumique plastique à partir de la déformation volumique totale mesurée (la déformation volumique élastique est calculée par le module k) ; ce qui permet d'obtenir les valeurs du paramètre $\mathrm{I}_{1}{ }^{*}$ et de $\mathrm{M} / \mathrm{K}$;

- dans la phase de cisaillement, au début, on considère l'état "critique " où le taux de déformation volumique plastique est nul, on détermine la pente M. En considérant l'état de contraintes correspondant à un écrouissage nul en contraintes, on détermine $\mathrm{M}^{*}$ : pente de la ligne d'écoulement et son ordonné à l'origine duquel on déduit le paramètre $\mathrm{c} / \mathrm{M}$.

Par le petit nombre de ses paramètres et l'hypothèse de plasticité associée, le modèle retenu se prête bien aux calculs numériques. Pour un modèle avec plasticité non associée, le choix d'un potentiel plastique conduit à faire intervenir d'autres paramètres, et les calculs numériques sont plus couteux ${ }^{(?)}$.

\section{4. ÉTUDE EXPÉRIMENTALE SUR LA GRAVE NON TRAITÉE 0/14}

Le comportement de ce matériau a fait l'objet d'une étude ${ }^{*}$ réalisée au moyen de l'équipement triaxial [3]. Les essais (consolidés-drainés) sont réalisés sur des échantillons cylindriques de diamètre égal à $10 \mathrm{~cm}$, et d'élancement égal à deux. A partir des résultats expérimentaux obtenus un bon calage des paramètres a été fait. Dans le tableau 1, (où $r$ désigne le coefficient de corrélation obtenu par une régression linéaire), on présente les valeurs obtenues sur trois échantillons. Par ailleurs, l'influence de différents types de trajets de charge sur les valeurs des paramètres a été testée. Ce qui a permis de vérifier le caractère intrinsèque des paramètres $\mathrm{M}^{*}$ et $\mathrm{c} / \mathrm{M}$ qui sont relatifs à l'écoulement de la grave. Leurs valeurs sont :

$$
\mathrm{M}^{*}=0,44 ; \mathrm{c} / \mathrm{M}=0,05 \mathrm{MPa}
$$

\section{SOLLICITATIONS ÉTUDIÉES}

Deux modes de chargement sont considérés. Pour une structure donnée et sur une partie de sa frontière, le premier mode consiste à imposer une contrainte (ou force) $Q$ qui schématise, par exemple, la surcharge d'un remblai sur le sol. Le deuxième mode consiste à imposer un déplacement $U$, qui peut simuler le cisaillement triaxial classique (en termes de vitesses), où on impose une vitesse de montée à l'échantillon. Il est à noter que le calcul avec déplacement imposé permet de suivre le comportement du matériau après la phase d'écrouissage positif [22] ;

\section{La formulation variationnelle avec le modèle «déplacement *}

Soit la structure $(\Omega)$, représentée sur la figure 7 , constituée par un matériau linéairement élastique. Sur sa frontière $\Gamma_{t}$ on impose soit le déplacement $U$, soit la contrainte $\mathrm{Q}$. Les forces de volume sont négligées. La formulation variationnelle, dans le cas déplacement imposé, s'obtient à partir des équations d'équilibre et des conditions aux limites. Soit l'espace des déplacements cinématiquement admissibles défini par:

\begin{tabular}{|c|c|c|c|}
\hline $\begin{array}{l}\text { Essais } \\
\text { Paramètres }\end{array}$ & TR1 & TR2 & TR4 \\
\hline$\sigma_{3}(\mathrm{MPa})$ & 0,123 & 0,092 & 0,062 \\
\hline$k(\mathrm{MPa})^{-1}$ & $0,75 \quad 10^{-4} \quad r=0,96$ & $0,6 \quad 10^{-4} \quad r=0,96$ & - \\
\hline $\mathrm{M} / \mathrm{K}$ & $0,35 \quad 10^{-2} \quad r=0,99$ & $0,72 \quad 10^{-2} \quad r=1,00$ & - \\
\hline $\mathrm{E}(\mathrm{MPa})$ & $75,1 \quad r=0,99$ & $64,5 \quad r=0,99$ & $r=0,97$ \\
\hline v & 0,22 & 0,29 & 0,14 \\
\hline $\mathrm{I}_{1}^{*}(\mathrm{MPa})$ & 0,072 & 0,062 & - \\
\hline$M$ & 0,18 & 0,17 & 0,17 \\
\hline $\mathrm{K}$ & 52 & 24 & - \\
\hline
\end{tabular}

Tableau 1. - Résultats de calage des paramètres du modèle à partir des essais triaxiaux réalisés.

Tableau 1. - Values of the model parameters derived from consolidated-drained triaxial tests.

( ) Le calcul des structures élastoplastiques avec Ihypothèse de plasticité non associée amène à la résolution de systèmes linéaires avec des matrices non symétriques. En plasticité associée ces matrices sont symétriques donc elles sont plus faciles à manipuler.

(*) Cette étude a été faite à IInstitut National des Sciences Appliquées de Rennes (France).

$$
V=\left\{v \text { *assez régulier *; } v \mid \Gamma_{t}=U \text {, et } v \mid \Gamma_{2}=0\right\} \text {. }
$$

Cette formulation, d'après [10], aboutit à l'équation (11) qui traduit le principe des puissances virtuelles 
appliqué à $(\Omega)$.

$$
\int_{\Omega} \sigma_{\mathrm{ij}} \varepsilon_{\mathrm{i}}(\mathrm{v}) \mathrm{d} \Omega=\mathrm{U}<\mathrm{q}>
$$

où $\langle\mathrm{q}\rangle$ est la contrainte moyenne définie par :

$$
\langle\mathrm{q}\rangle=\int_{r, \sigma_{\mathrm{g}} \mathrm{n} \mathrm{d} \Gamma \mathrm{r}}
$$

D'une manière analogue à la précédente on obtient pour le cas contrainte imposée la formulation variationnelle qui se traduit par l'équation:

$$
\int_{\Omega} \sigma_{i j} \varepsilon_{i j}(v) d \Omega=Q<u>
$$

où $<\mathrm{u}>$ représente le déplacement moyen, qui est donné par :

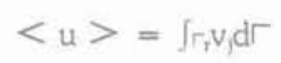

L'étude de ce problème par la méthode des éléments finis, pour le cas contrainte imposée ou déplacement imposé, se ramène à résoudre le système linéaire [10] :

$$
\mathrm{AU}=\mathrm{L}
$$

où :

A est la matrice de rigidité globale de la structure,

L est le vecteur forces généralisées aux nœuds chargés, $\mathrm{U}$ est le vecteur déplacements aux nœeuds de la structure.

\section{RÉSULTATS NUMÉRIQUES OBTENUS}

\subsection{Mise en œuvre numérique du modèle retenu}

Elle a été faite par la méthode des éléments finis avec le modèle "déplacement ». Deux buts sont recherchés :

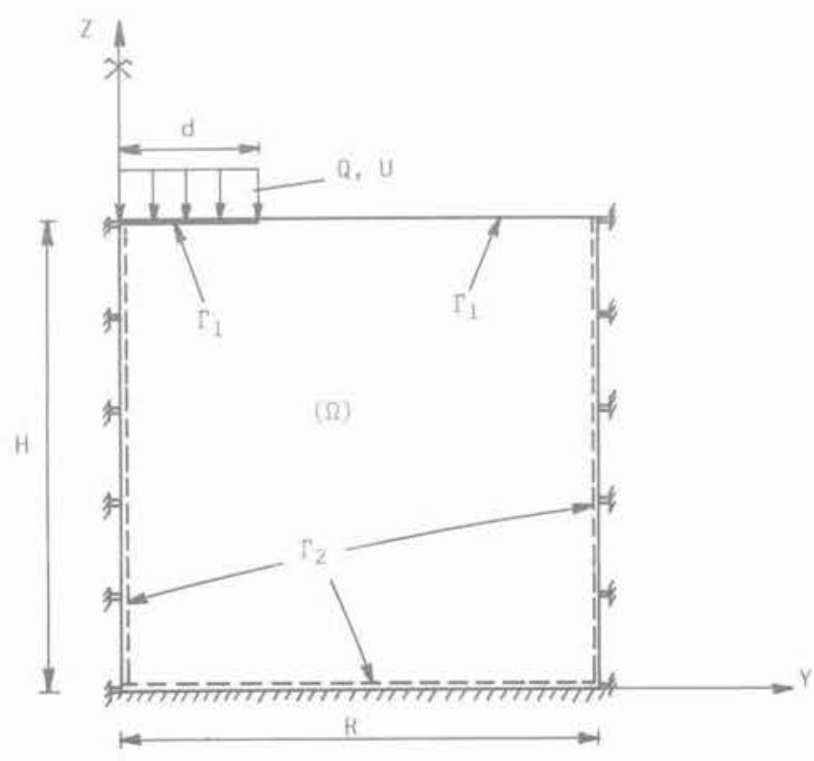

Fig. 7. - La structure ( $\Omega$ ) avec ses frontières et conditions sux limites.

Fig. 7. - Boundary conditions for the structure $\Omega$ ). la simulation des essais réalisés moyennant les valeurs des paramètres obtenues après calage; et le calcul de structures en élastoplasticité où deux exemples sont traités.

La formulation variationnelle du problème d'évolution d'une structure élastoplastique, avec l'hypothèse de charge $e^{(*)}$, conduit à la résolution d'un système non linéaire [3], qui est ramené à résoudre une suite de problèmes linéaires qui s'écrivent sous la forme suivante:

$$
\left(A-A^{*}\left(U^{(n-1)}\right)\right) \dot{U}(n)=\dot{L}^{(n)}
$$

où A est la même matrice de rigidité globale utilisée dans (15), $A^{*}$ est la matrice déterminée en fonction des termes du tenseur d'élastoplasticité $\mathbb{E}^{\left.\mathrm{p}^{\left({ }^{\prime}-\right)}\right)}$ à partir des matrices de rigidité élémentaire des éléments plastifiés; pour ces derniers l'état de contraintes vérifie les conditions (3a), (3b), et (3c).

$\dot{U}^{(n)}$ et $\dot{L}^{(n)}$ sont respectivement les vecteurs incréments des déplacements et des forces généralisées aux nœuds obtenus à l'incrément (n) de la charge.

\subsection{Simulation des essais}

Un programme de calcul a été élaboré pour la simulation des essais triaxiaux où divers types de trajets de charge peuvent être envisagés [3]. On donne quelques résultats relatifs à l'essai triaxial classique.

Sur les figures 8 et 9 on note une concordance parfaite entre les résultats expérimentaux et numériques pour les déformations volumiques plastique et totale. En effet, en consolidation isotrope le calage des paramètres est obtenu avec de bons coefficients de corrélation (tableau 1). Dans la phase de cisaillement, pour les déformations volumiques totale (fig. 10) et plastique (fig. 11), la concordance est acceptable pour les petites déformations. Les résultats numériques surestiment la dilatance en grandes déformations. La comparaison entre la figure 10 et la figure 11 montre que l'état * critique» $\left(\dot{e}_{1}^{p}=0\right)$ a lieu à un niveau de contraintes inférieur à celui de l'état caractéristique $\left(\dot{e}_{1}=0\right)$. On remarque sur la figure 12 que les calculs numériques permettent d'atteindre le déviateur maximum, obtenu par l'essai, avec une concordance assez bonne. On note, en plus, que le calcul avec déplacement imposé permet de bien simuler le palier de la courbe effortdéformation mieux que le calcul avec contrainte imposée. Cependant la partie décroissante de la courbe (qui correspond à l'écrouissage négatif) n'a pu être obtenue avec l'algorithme utilisé. Concernant ce point, un travail intéressant a été fait par KALTEZIOTIS et al. (1984) sur la correction de la capacité portante des argiles saturées, en se basant sur la technique proposée par TANZI et al. (1982).

Du point de vue numérique les calculs avec contrainte et déplacement imposés sont identiques, sauf en grandes déformations, et avec déplacement imposé, on peut mieux suivre le comportement du matériau. A la lumière de ces résultats, le modèle retenu simule bien le comportement observé surtout en petites déformations où la majorité des paramètres sont calés.

$\left({ }^{\circ}\right) \mathbb{E}^{D}=\left(\mathbb{E},\left(\delta \mathrm{F}(\delta \sigma)^{\prime} \cdot(\delta \mathrm{F} / \delta \sigma) \cdot \mathbb{E}\right) / \mathrm{h}_{\varepsilon}\right.$.

(“) Le cas de la décharge n'est pas considéré. 


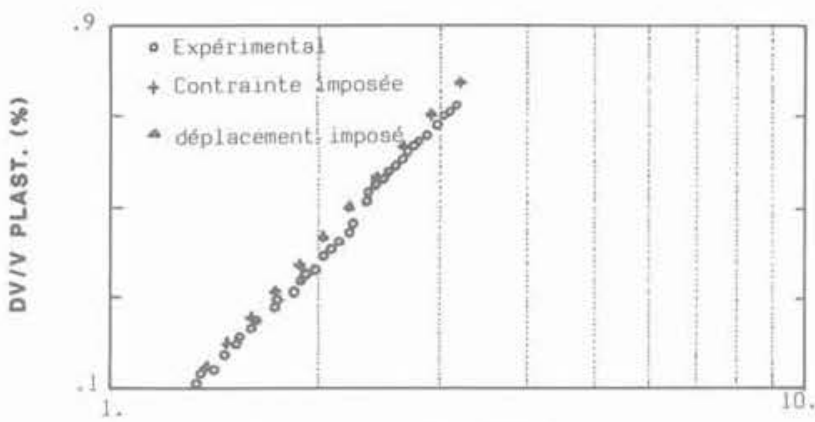

(b) TR2- $(11+c / M)(M P a E-01)$

Fig. 8. - Evolution de la déformation volumique plastique dans une consolidation isotrope.

Fig. 8. - Variation of plastic volumic strain with stress in isotropic consolidation.

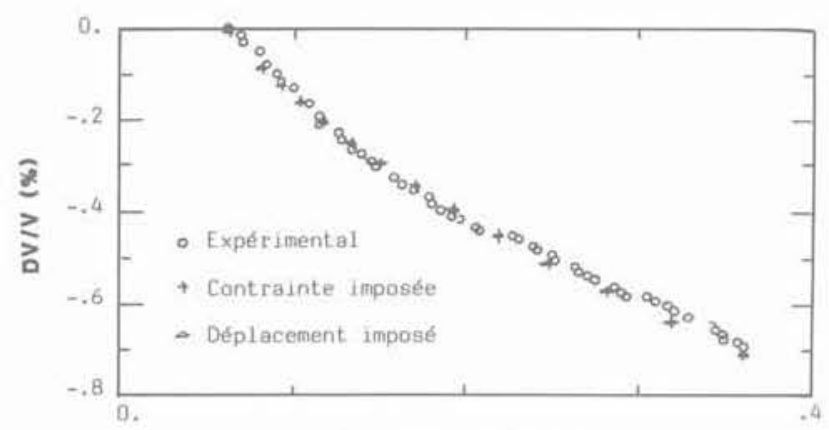

(a) TR1- 11 (MPa)

Fig. 9. - Evolution de la déformation volumique totale dans une consolidation isotrope.

Fig. 9. - Variation of total volumic strain with stress in isotropic consolidation.

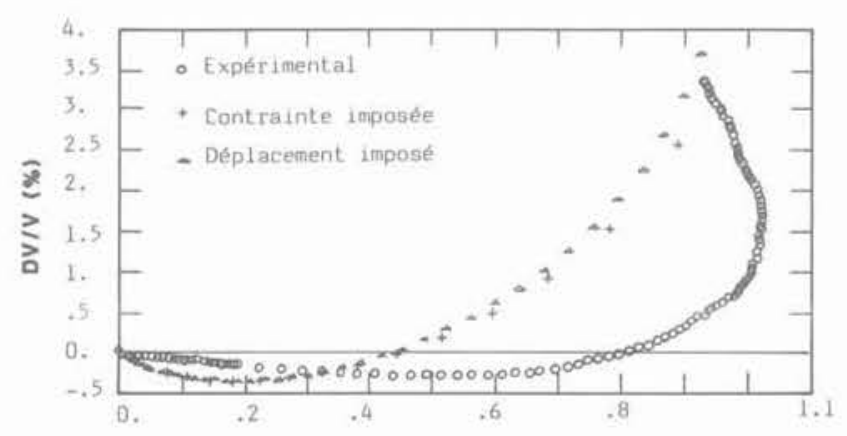

(b) TR2- 9 (MPa)

Fig. 10. - Evolution de la déformation volumique totale dans un cisaillement triaxial.

Fig, 10. - Variation of total volumic strain with deviatoric stress in triaxial shear.

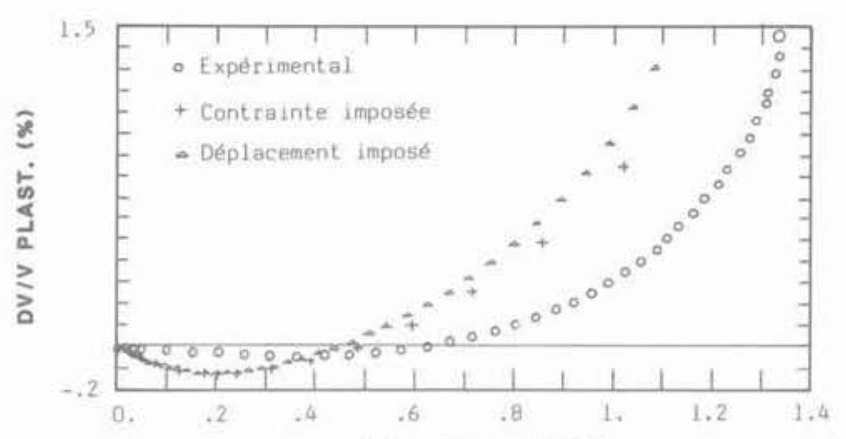

(a) TR1- $q(M P a)$

Fig. 11. - Evolution de la déformation volumique plastique dans un cisaillement triaxial.

Fig. 11. - Variation of plastic volumic strain with stress in triaxial shear.

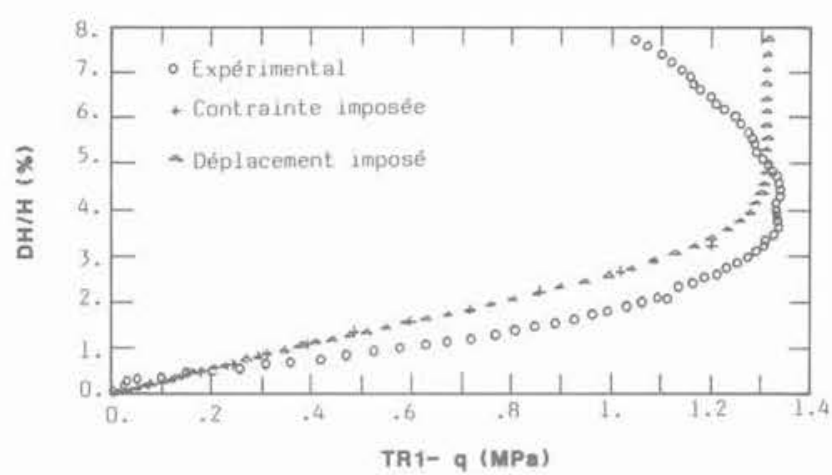

Fig. 12. - Evolution de la déformation axiale dans un cisaillement triaxial.

Fig. 12. - Variation of axial strain with stress in triaxial shear.

\section{CALCUL DES STRUCTURES}

Deux exemples de structures axisymétriques sont traités : le premier est celui d'un massif homogène en grave, le second est celui d'une chaussée souple à base du même matériau (fig. 13). Pour ces deux cas on utilise la même schématisation représentée sur la figure 7 ; avec :

$$
\mathrm{R}=70 \mathrm{~cm}, \mathrm{H}=80 \mathrm{~cm}, \mathrm{~d}=12 \mathrm{~cm} .
$$

Le maillage est constitué de 132 éléments rectangulaires à 4 noeuds.

Le comportement de la grave est régi par le modèle retenu, avec les caractéristiques suivantes :

- en élasticité :

$$
\mathrm{E}_{2}=75,1 \mathrm{MPa} ; \nu_{2}=0,22 ;
$$


- en plasticité :

$$
\begin{gathered}
\mathrm{M}=0,18 ; \mathrm{K}=52,0 ; \mathrm{I}_{1}^{*}=0,072 \mathrm{MPa} ; \\
\mathrm{M}^{*}=0,44 ; \mathrm{c} / \mathrm{M}=0,05 \mathrm{MPa} ; \\
\mathrm{C}^{\prime}=0,03 \mathrm{MPa} ; \varphi^{\prime}=55^{\circ} .
\end{gathered}
$$

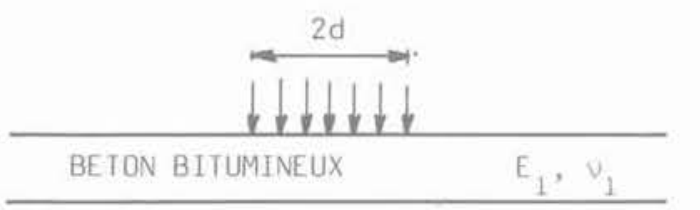

$$
\text { GRAVE NON TRAITEE } 0 / 14 \quad E_{2}, v_{2}
$$

SOL DE FONDATION

$$
E_{3}, v_{3}
$$

Fig. 13, - Un exemple de chaussée souple. Fig. 13. - An example of flexible pavement.

Le béton bitumineux et le sol de fondation (fig. 14) sont considérés comme étant linéairement élastiques. Leurs caractéristiques sont :

$$
\begin{gathered}
\mathrm{E}_{1}=5000 \mathrm{MPa} ; v_{1}=0,33 \\
\mathrm{E}_{3}=40 \mathrm{MPa} ; v_{3}=0,33 .
\end{gathered}
$$

La résolution est faite par intégration numérique [7]. Le chargement (avec contrainte ou déplacement imposé) est fait par incréments, quil sont calculés de manière que la structure se plastifie élément par élément. Les conditions de la charge plastique sont vérifiées à chaque incrément [3].

\subsection{Cas du massif en grave non traitée}

On cherche à déterminer la valeur de la charge $Q$ qu'on peut appliquer sur le massif, avec un chargement en contrainte ou déplacement imposé. Les calculs sont conduits jusqu'à ce que l'hypothèse de la charge plastique ne soit plus vérifiée : le multiplicateur $\dot{\lambda}$ (en déformations) est négatif. En utilisant la méthode de résolution décrite dans 6.1 , et les hypothèses précédentes, on détermine deux valeurs maximales de la charge. Elles sont respectivement égales avec contrainte imposée et déplacement imposé :

$$
\mathrm{q}_{\max }^{\mathrm{Q}}=0,158 \mathrm{MPa} ; \mathrm{q}_{\max }^{\mathrm{U}}=0,048 \mathrm{MPa} \text {. }
$$

Ces valeurs peuvent être améliorées en envisageant le cas de décharge, dans l'algorithme utilisé. Avec déplacement imposé la charge maximale représente $30 \%$ de la valeur obtenue avec contrainte imposée.

L'évolution de la zone plastifiée se ressemble pour les deux modes de chargement (fig. 14 et 15). Ce n'est pas le cas pour la zone de dilatance (fig. 16 et 17), avec déplacement imposé cette zone ne concerne que la verticale au bord du disque de chargement; avec contrainte imposée cette zone atteint l'axe de la charge. On

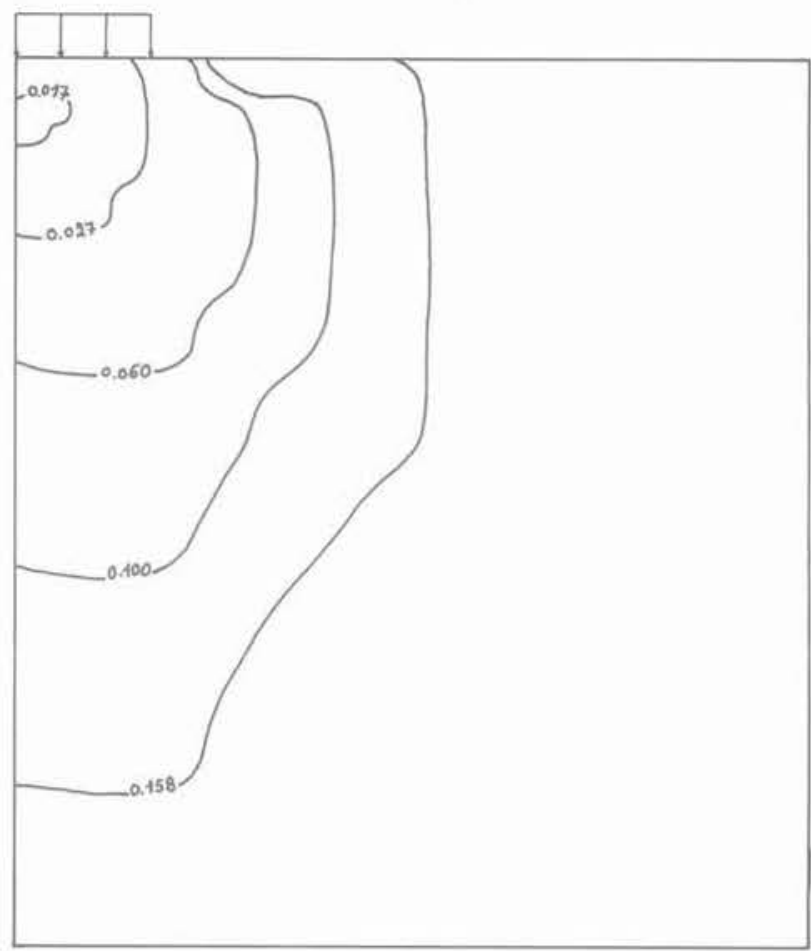

Fig. 14. - Evolution de la zone plastifiée en fonction du chargement avec contrainte imposée (MPa).

Fig. 14. - Evolution of the plastified area for an imposed stress loading.

\section{Massif en grave}

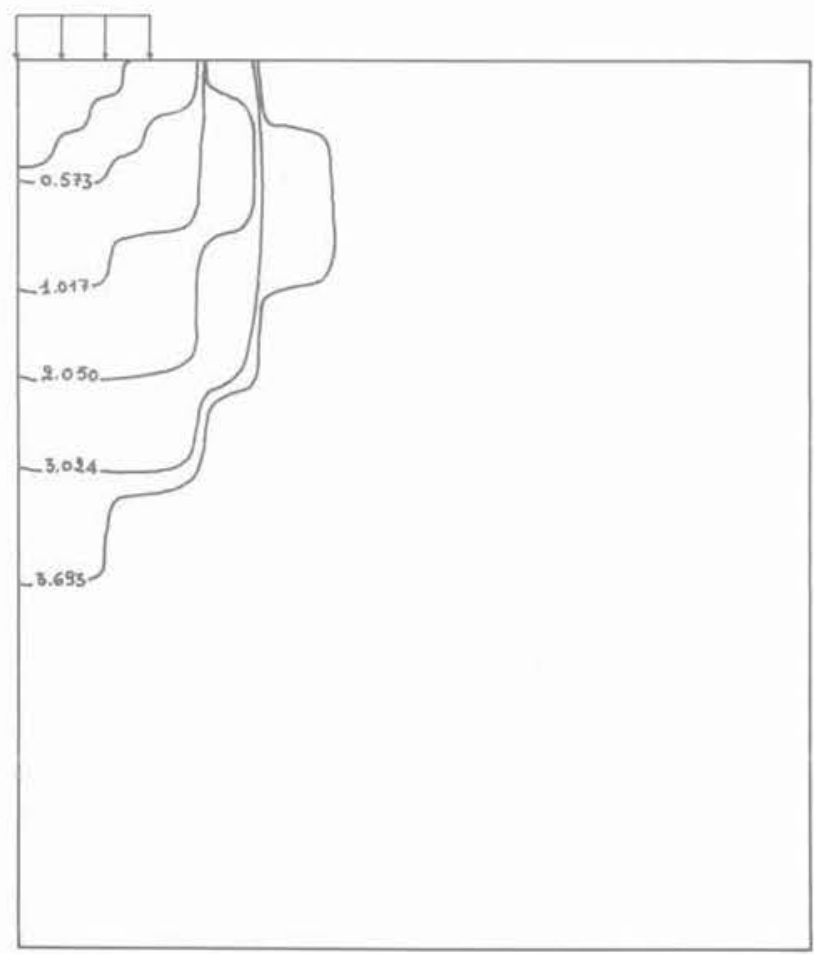

Fig. 15. - Evvolution de la zone plastifiée en fonction du chargement avec déplacement imposé $\left(10^{-2} \mathrm{~cm}\right)$. Fig. 15. - Evolution of the plastified area for an imposed displacement loading. 
Massif en grave

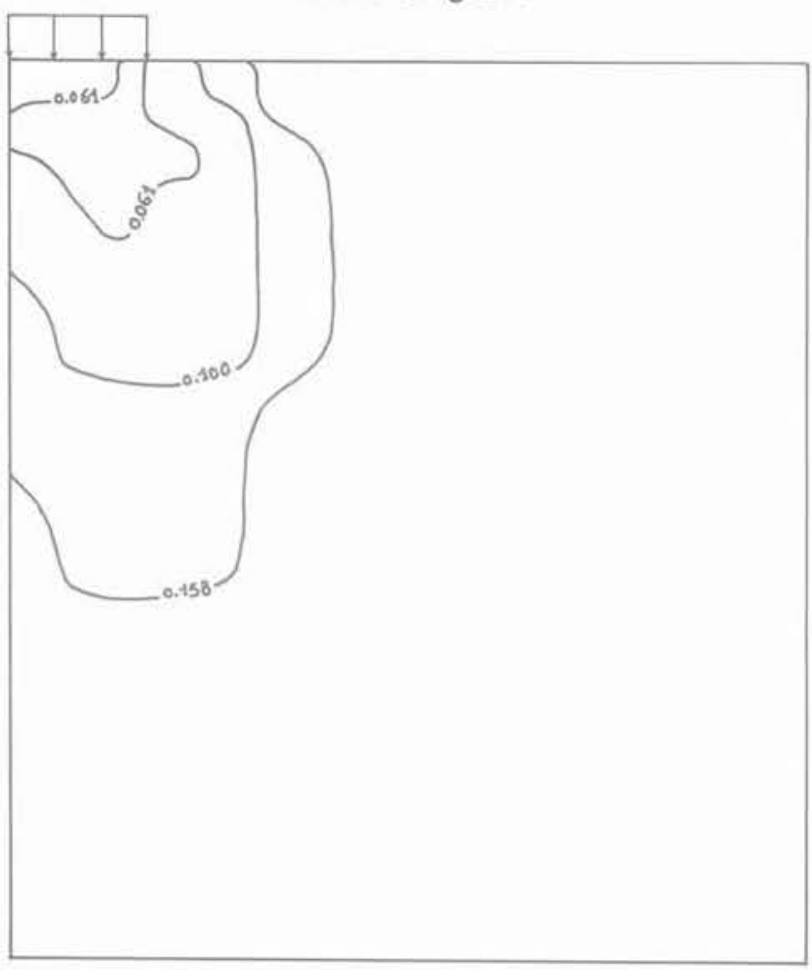

Fig. 16. - Evolution de la zone de dilatance en fonction du chargement (contrainte imposée en MPa).

Fig. 16. - Evolution of the dilatancy area for an imposed stress loading.

\section{Massif en grave}

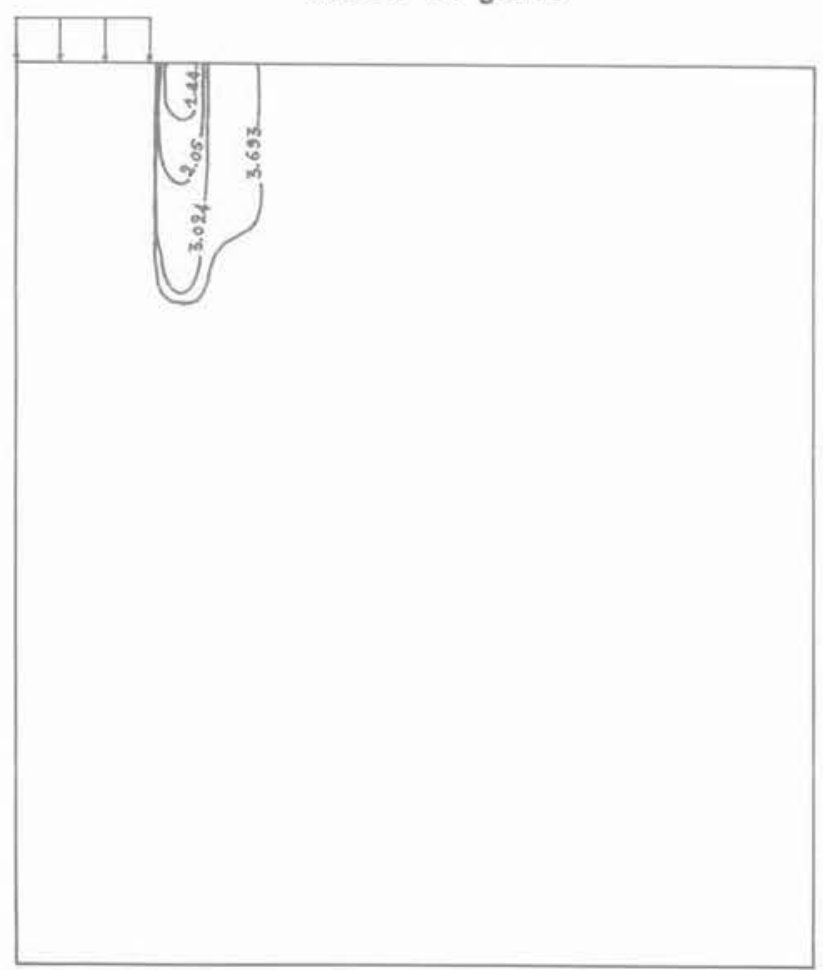

Fig. 17. - Evolution de la zone de dilatance en fonction du chargement avec déplacement imposé $\left(10^{-2} \mathrm{~cm}\right)$.

Fig. 17. - Evolution of the dilatancy area for an imposed displacement loading. note que les zones de comportement obtenues avec déplacement imposé sont plus restreintes que celles obtenues avec contrainte imposée (fig. 18 et 19). Elles sont situées au bord du disque de chargement.

Les répartitions de contraintes montrent dans ce cas : - pour la contrainte radiale (fig. 20 et 21 ), le modèle retenu donne des contraintes en compression, or qu'en élasticité linéaire des contraintes de traction apparaissent; elles sont plus marquées avec déplacement imposé;

- pour la contrainte verticale (fig. 22 et 23), les répartitions diffèrent. Avec contrainte imposée la différence entre les deux modèles est petite en surface; avec déplacement imposé les valeurs sont faibles en élasticité;

- pour la contrainte de cisaillement (fig. 24 et 25), avec contrainte ou déplacement imposé, le modèle donne de très grandes valeurs au bord du disque de chargement. Elles sont à l'origine de la « ruine » des éléments en état d'écrouissage négatif qui est développé dans la même zone.

Il est à noter qu'avec déplacement imposé, les contraintes dépassent la charge maximale, ce quil n'est pas vrai avec contrainte imposée.

Masslf on grave

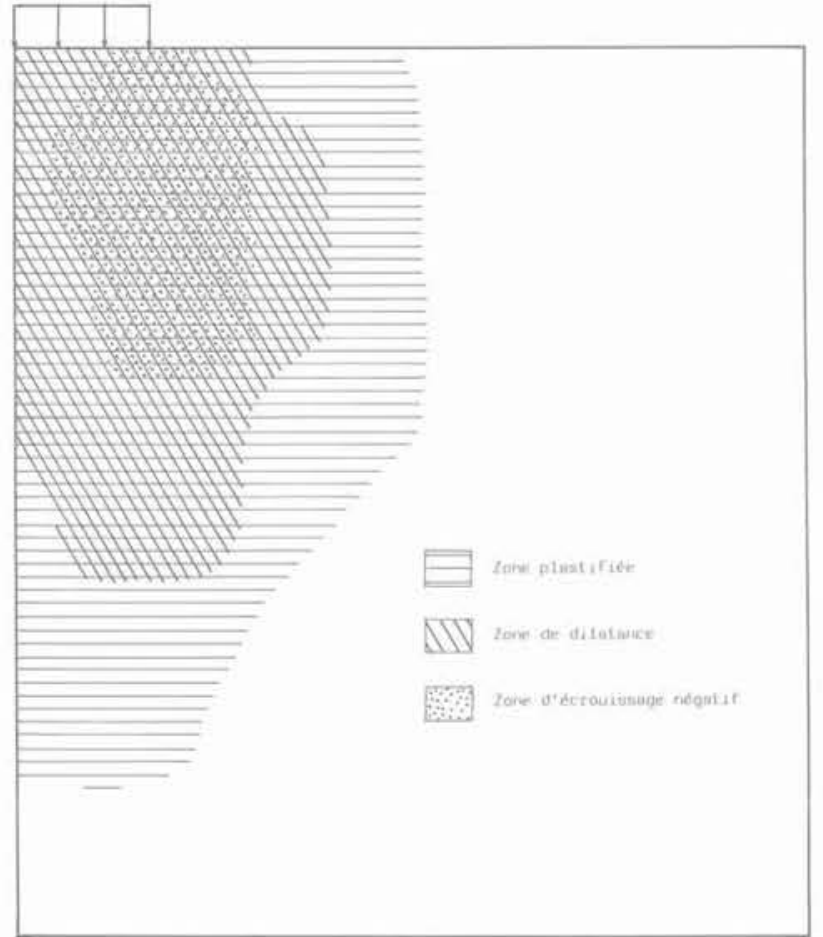

Fig. 18. - Limites des différentes zones de comportement obtenues pour la charge maximale (calcul avec contrainte imposée).

Fig. 18. - Limits of the differents behaviour areas obtained at the maximum load (imposed stress computation). 
Massif en grave
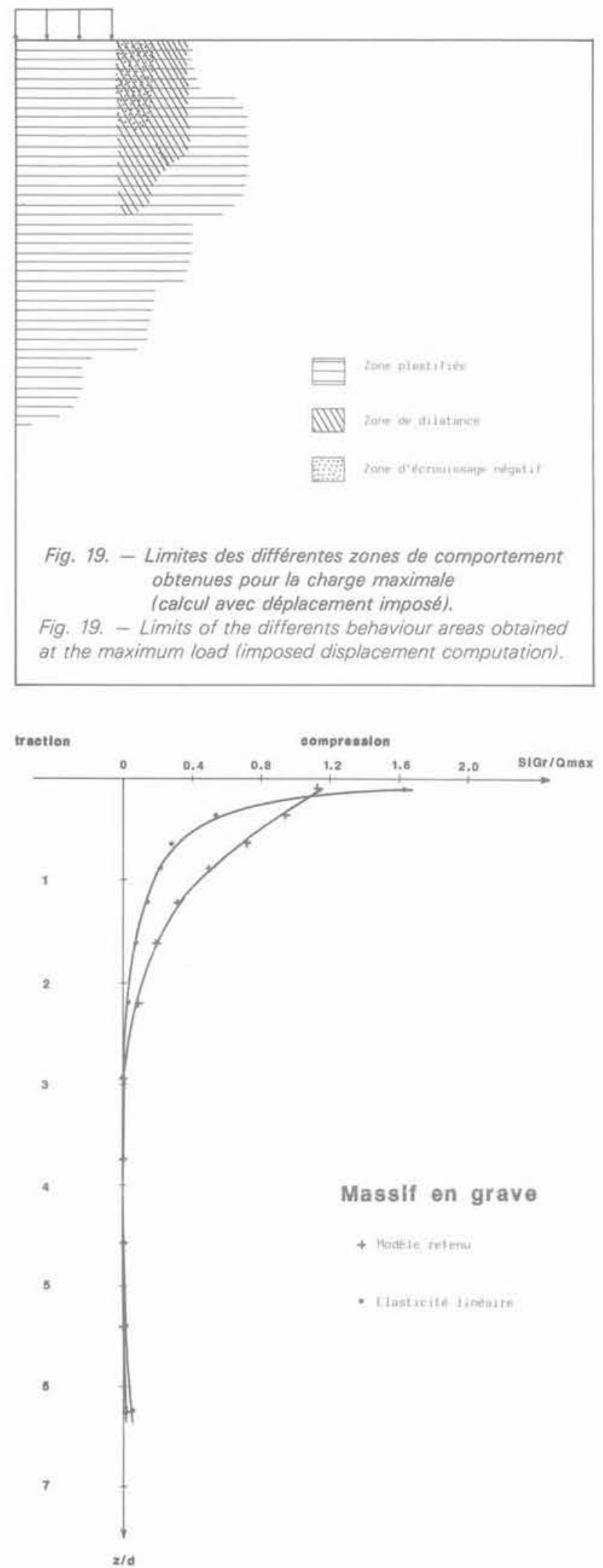

Fig. 20. - Evolution de la contrainte radiale sous l'axe de la charge (calcul avec déplacement imposée).

Fig. 20. - Variation of radial stress, under the load axis, with depth limposed displacement computationl.

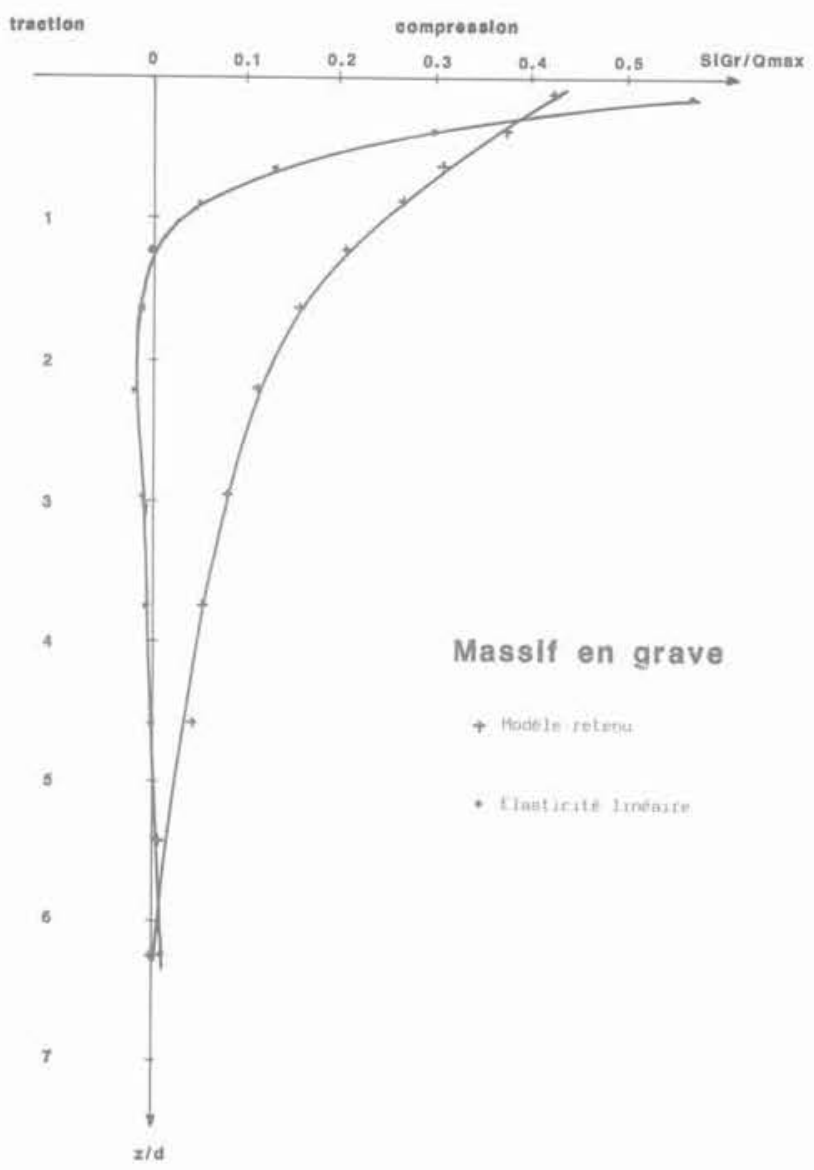

Fig. 21. - Evolution de la contrainte radiale sous l'axe de la charge (calcul avec contrainte imposée).

Fig. 21. - Variation of radial stress, under the load axis, with depth limposed stress computation).

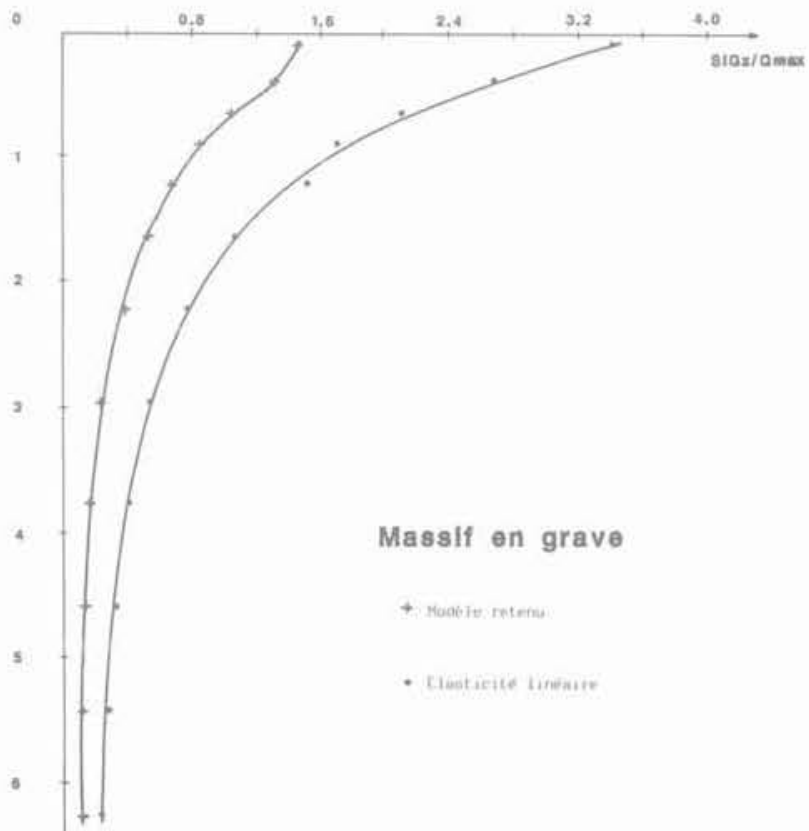

Fig. 22. - Evolution de la contrainte verticale sous l'axe de la charge (calcul avec déplacement imposé).

Fig. 22. - Variation of vertical stress, under the load axis, with depth limposed displacement computation). 

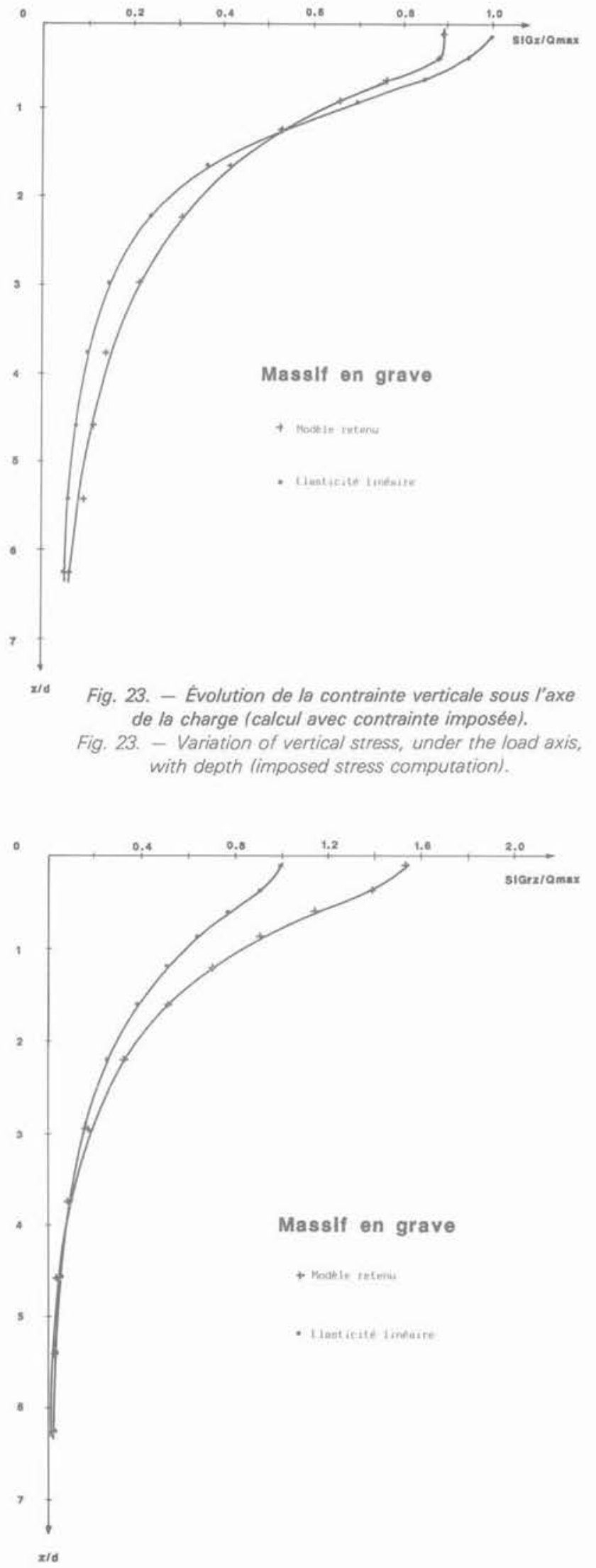

Fig. 24. - Evolution de la contrainte de cisaillement au bord du disque de chargement (calcul avec déplacement imposé). Fig. 24. - Variation of shear stress, at the load edge, with depth (imposed displacement computation).

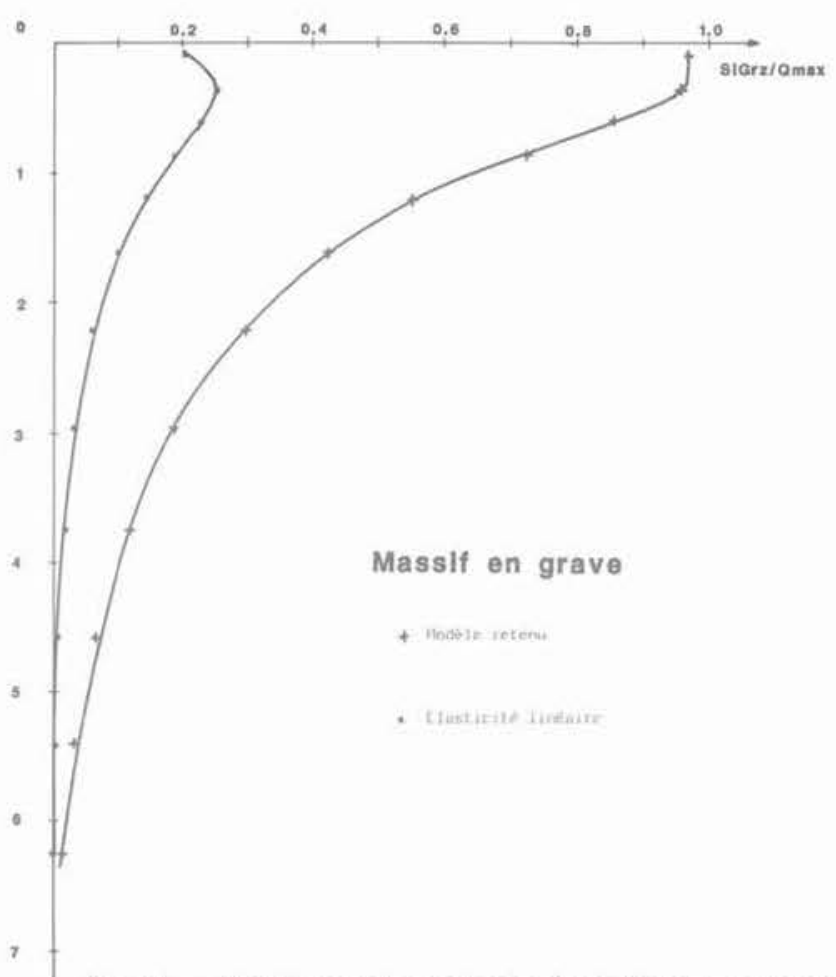

Fig. 25. - Evolution de la contrainte de cisaillement au bord z/d du disque de chargement (calcul avec contrainte imposée).

Fig. 25. - Variation of shear stress, at the load edge, with depth (imposed stress computation).

\subsection{Cas de la chaussée souple}

Pour simuler l'action de la roue d'un véhicule sur la chaussée le chargement avec contrainte imposée a été retenu; pour lequel les calculs ont été conduits jusqu'à la valeur $\mathrm{Q}=0,9 \mathrm{MPa}$. L'écrouissage négatif n'apparaît pas dans la couche de la grave. La couche de béton bitumineux joue le rôle d'une dalle de répartition de la charge, qu'on peut voir par l'évolution de la zone plastifiée (fig. 26). La zone de dilatance (fig. 27) prend naissance au bord du disque de chargement sans atteindre l'axe de la charge. Pour la contrainte radiale, le modèle ne donne pas de contraintes de traction comme en élasticité linéaire (fig. 28). L'augmentation en profondeur, de cette contrainte, pourrait être attribuée à l'état de dilatance dans les éléments situés au niveau du bord du disque de chargement. Ces derniers exercent une compression sur les éléments (en état contractant) situés sous l'axe de la charge. L'évolution du déplacement vertical en surface (fig. 29), montre qu'avec le modèle retenu les valeurs obtenues sont élevées : cela est dû à la plastification des éléments en grave sous le béton bitumineux.

\section{3. Étude comparative des méthodes de calcul}

Elle a pour but de comparer, sur le cas du massif homogène en grave, les méthodes de calculs au niveau des résultats, et leur donner les conditions nécessaires pour une mise en application correcte concernant le choix de la valeur de l'incrément de la charge.

La détermination de la charge maximale par les deux méthodes a été étudiée d'une part en faisant varier 
Chaussée souple

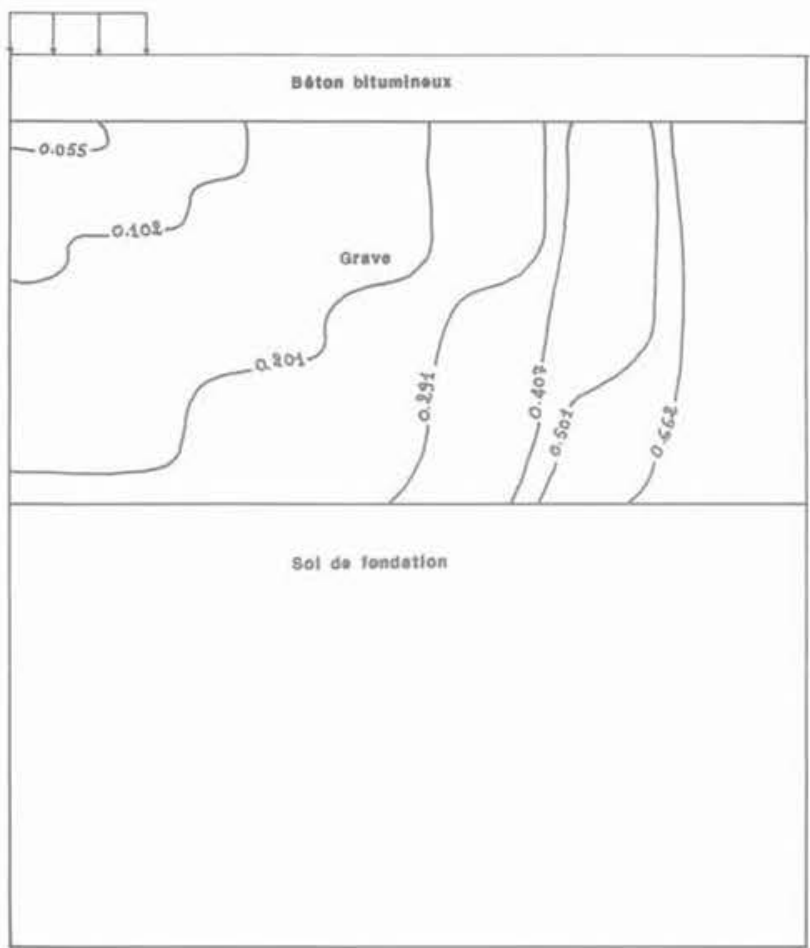

Fig. 26. - Evolution de la zone plastifiée en fonction du chargement (contrainte imposée en MPa).

Fig. 26. - Variation of the plastified area with an imposed stress loading.

\section{Chaussée souple}

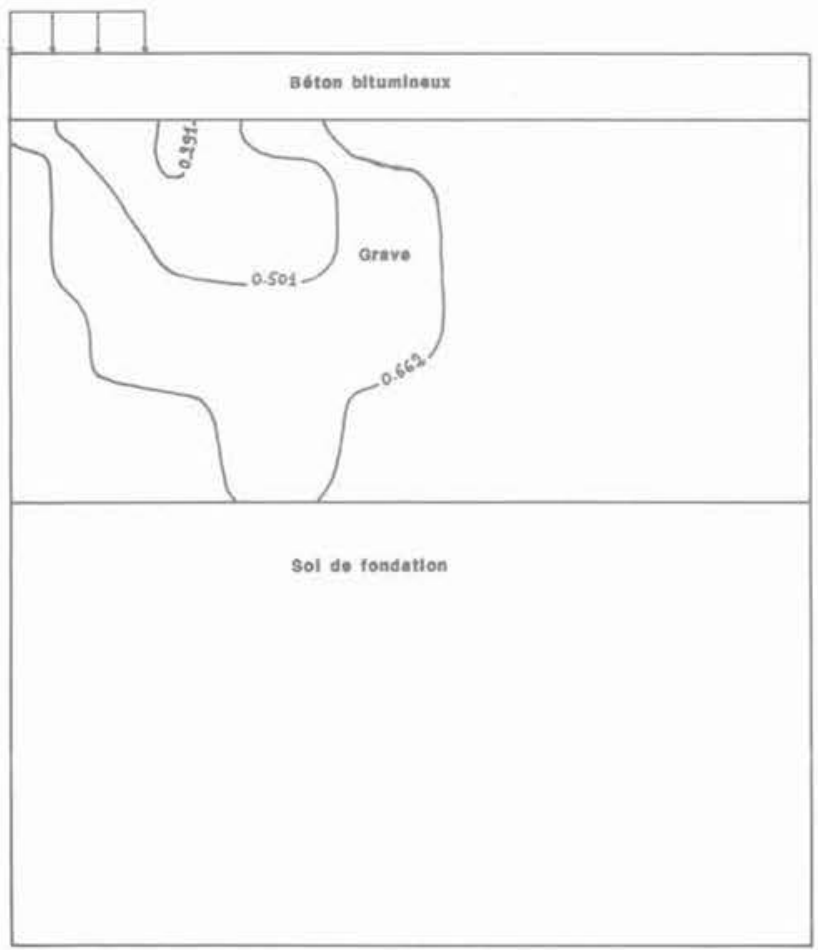

Fig. 27. - Evolution de la zone de dilatance en fonction du chargement (contrainte imposée en $\mathrm{MPa}$ ).

Fig. 27. - Variation of the dilatancy area with an imposed stress loading.

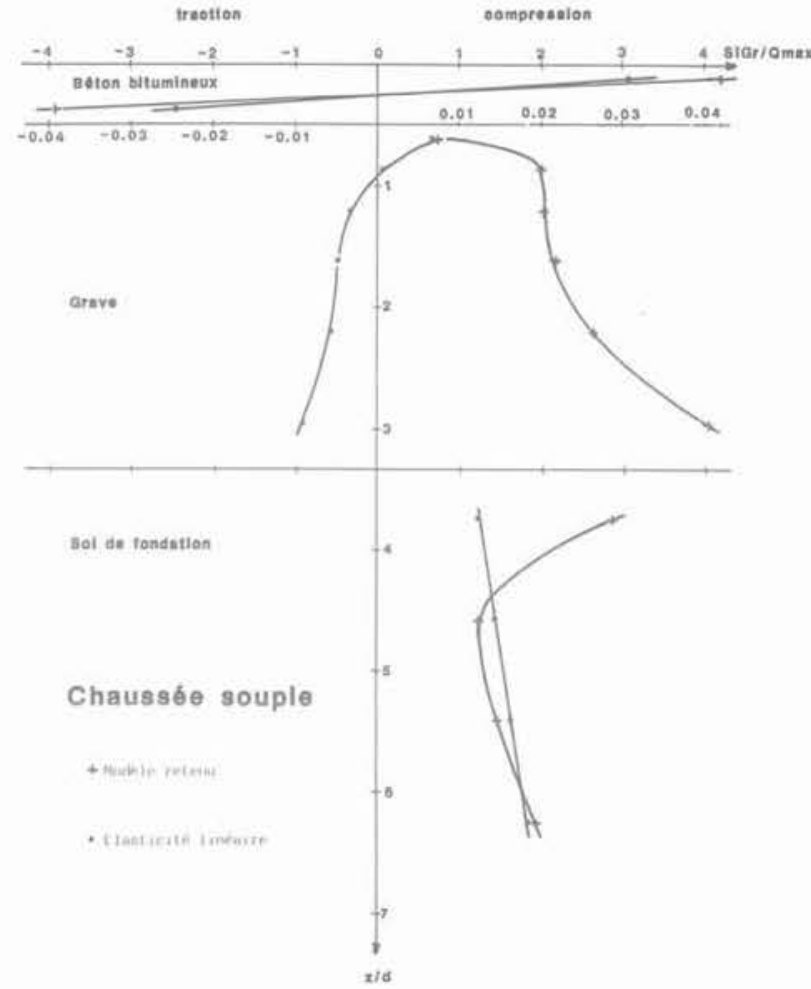

Fig. 28. - Evolution de la contrainte radiale sous l'axe de la charge (calcul avec contrainte imposée).

Fig. 28. - Variation of radial stress, under the load axis, with depth limposed stress computation).

l'incrément maximum de la charge, d'autre part en considérant un deuxième maillage $n^{\circ} 2$, plus raffiné que le premier $n^{\circ} 1$, au bord du disque de chargement. Cette façon permet d'avoir des valeurs plus précises sur les contraintes qui sont élevées dans cette zone ; et par suite une meilleure estimation de la charge maximale.

Les résultats obtenus (voir tableaux 2 et 3 ), permettent de noter :

- avec le maillage $n^{\circ} 1$, en doublant la valeur de l'incrément maximum on aboutit à une légère augmentation de la charge maximale ( $7 \%$ au plus) ;

- avec le maillage $n^{\circ} 2$ avec déplacement imposé, en doublant l'incrément maximum on obtient une nette amélioration de la charge maximale $(39,4 \%)$. Mais sa valeur est inférieure à celle obtenue par le maillage $n^{\circ} 1$;

- avec contrainte imposée, l'augmentation de l'incrément maximum n'est pas avantageuse, elle aboutit à une diminution de la charge maximale;

- l'augmentation de l'incrément maximum, au-delà du double, entraîne une diminution du nombre d'itérations de calcul; mais l'hypothèse de la charge plastique 


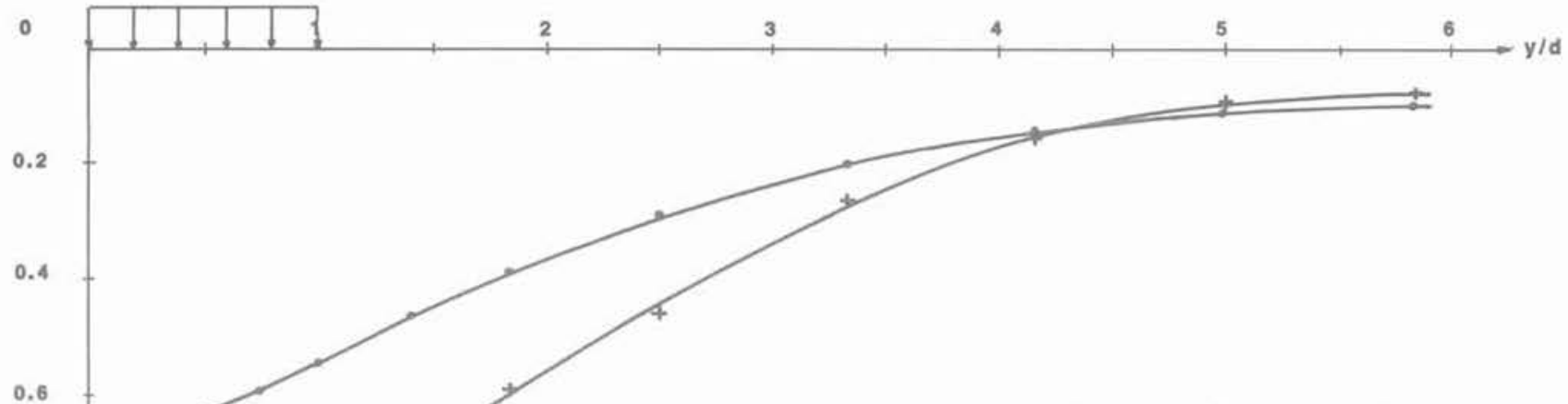

\section{Chaussee souple}

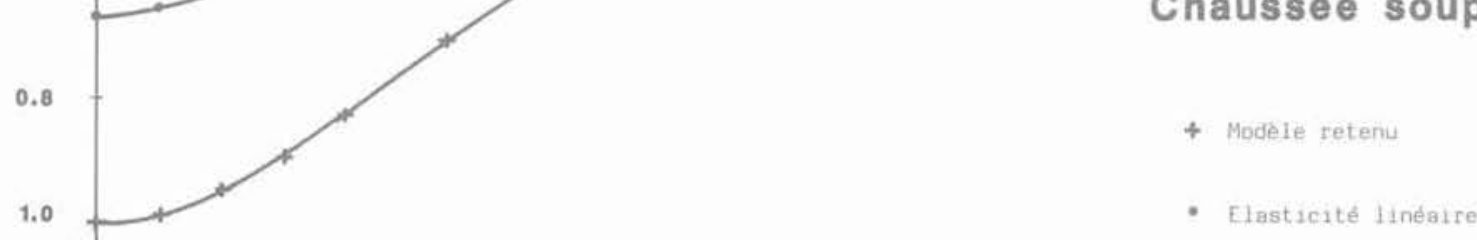

Fig, 29, - Evolution du déplacement vertical en surface $(z=0)$

pour la charge $Q=0,662 \mathrm{MPa}$ (contrainte imposée).

Fig. 29. - Variation of vertical displacement with radius

(null depth) for the load value $Q=0.662 \mathrm{MPa}$ (imposed stress

computationl.

n'est plus vérifiée. Les résultats trouvés sont traduits sur les figures 30 et 31 . L'incrément maximum de la charge ne doit pas dépasser les valeurs suivantes pour les chargements avec contrainte imposée et avec déplacement imposé :

$$
\Delta \mathrm{Q}=0,02 \mathrm{MPa} ; \Delta \mathrm{U}=0,002 \mathrm{~cm} .
$$

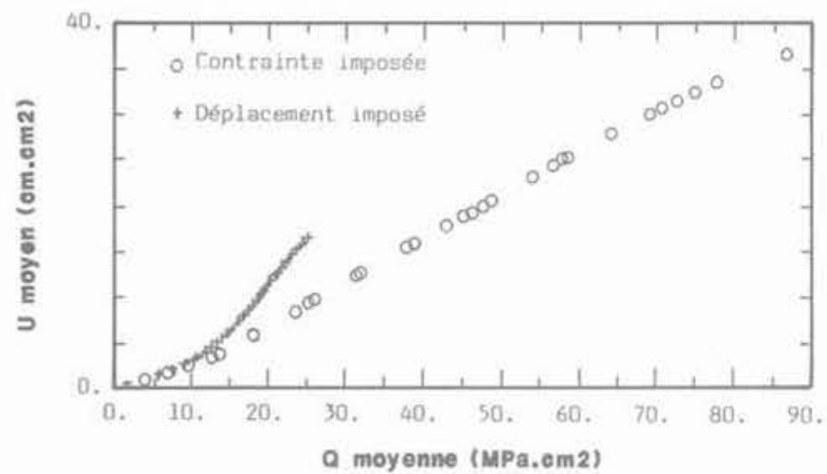

Fig. 30. - Contrainte moyenne obtenue avec le maillage $n^{\circ} 1$ (incrément maximum unitaire).

Fig. 30. - The mean stress determined with mesh $n^{\circ} 1$ (unitary maximum load).

\section{CONCLUSION}

A partir des phénomènes expérimentaux observés sur la grave, on justifie le choix d'un modèle élastoplastique avec écrouissage pour ce matériau.

Ce modèle tient compte de la variation de volume avant et au cours de l'écoulement. On y fait intervenir

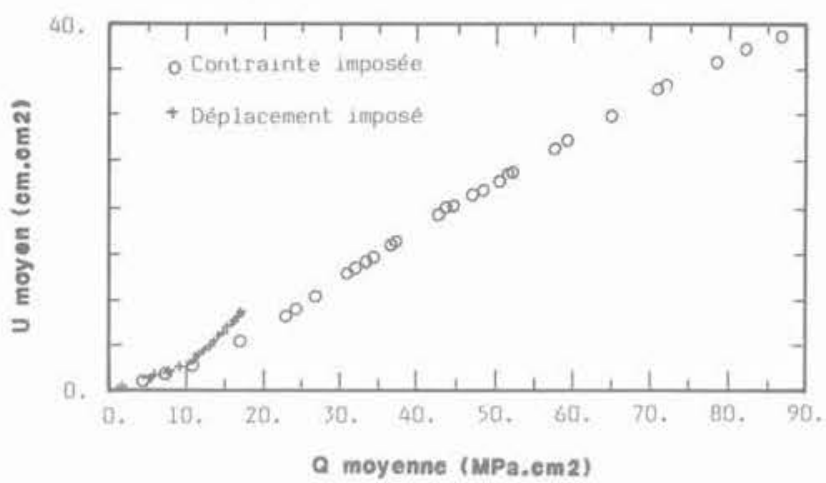

Fig. 31. - Contrainte moyenne obtenue avec le maillage $n^{\circ} 2$ (incrément maximum unitaire)

Fig. 31. - The mean stress determined with mesh $n^{\circ} 2$ (unitary maximum load).

cinq paramètres qu'on détermine par un essai triaxial classique.

Lors d'une étude expérimentale sur une grave non traitée $0 / 14$, un calage des paramètres a permis de simuler les essais réalisés. On vérifie que le modèle décrit bien les phénomènes observés en petites déformations.

En considérant les chargements contrainte imposée et déplacement imposé, on vérifie que le deuxième mode de chargement permet de mieux simuler le comportement du matériau en grandes déformations. Le problème de simulation de l'écrouissage négatif qui régit le comportement du matériau après écoulement reste encore ouvert.

Deux exemples d'application ont été traités avec le modèle retenu. Le calcul d'un massif homogène en grave a permis de déterminer une valeur maximale de la charge. Le calcul d'une chaussée souple, à base de 


\begin{tabular}{|c|c|c|c|c|c|c|c|c|c|}
\hline \multicolumn{2}{|c|}{$\begin{array}{l}\text { Incrément } \\
\text { max. } \\
\text { Maillage }\end{array}$} & \multicolumn{2}{|c|}{0,01} & \multicolumn{2}{|c|}{0,02} & \multicolumn{2}{|l|}{0,04} & \multicolumn{2}{|c|}{0,08} \\
\hline \multirow{2}{*}{1} & $\begin{array}{l}\text { Contrainte } \\
\text { moyenne } \\
\left(\mathrm{Mpa} . \mathrm{cm}^{2}\right)\end{array}$ & 86,9 & (32) & 93,2 & (24) & & * & & * \\
\hline & $\begin{array}{l}\text { déplacement } \\
\text { moyen } \\
\left(\mathrm{cm} \cdot \mathrm{cm}^{2}\right)\end{array}$ & 36,7 & (32) & 38,6 & (24) & & " & & * \\
\hline \multirow{2}{*}{2} & $\begin{array}{l}\text { contrainte } \\
\text { moyenne } \\
\text { (MPa. } \mathrm{cm}^{2} \text { ) }\end{array}$ & 86,7 & (33) & 17,2 & * & 17,2 & * & 11,2 & * \\
\hline & $\begin{array}{l}\text { déplacement } \\
\text { moyen } \\
\left(\mathrm{cm} \mathrm{cm}^{2}\right)\end{array}$ & 38,4 & (33) & 7,8 & $*$ & 7,8 & * & 2,73 & * \\
\hline
\end{tabular}

Tableau II. - Contrainte et déplacement moyens obtenus pour la charge maximale; calcul avec contrainte imposée.

Tableau II. - The mean values of stress and displacement obtained at the maximum load; imposed stress computation.

\begin{tabular}{|c|c|c|c|c|c|c|c|}
\hline \multicolumn{2}{|c|}{$\begin{array}{l}\text { Incrément } \\
\text { max. } \\
\text { Maillage }\end{array}$} & \multicolumn{2}{|c|}{0,001} & \multicolumn{2}{|c|}{0,002} & 0,004 & 0,008 \\
\hline \multirow{2}{*}{1} & $\begin{array}{l}\text { Contrainte } \\
\text { moyenne } \\
(\text { Mpa.cm²) }\end{array}$ & 25,1 & (50) & 26,7 & (35) & & * \\
\hline & $\begin{array}{l}\text { déplacement } \\
\text { moyen } \\
\left(\mathrm{cm} . \mathrm{cm}^{2}\right)\end{array}$ & 16,7 & (50) & 17,3 & (35) & & * \\
\hline \multirow{2}{*}{2} & $\begin{array}{l}\text { contrainte } \\
\text { moyenne } \\
\left(\mathrm{MPa} . \mathrm{cm}^{2}\right)\end{array}$ & 17,0 & (29) & 23,6 & (31) & 20,3 & * \\
\hline & $\begin{array}{l}\text { déplacement } \\
\text { moyen } \\
\left(\mathrm{cm} \cdot \mathrm{cm}^{2}\right)\end{array}$ & 8,5 & (29) & 15,2 & (31) & 9,1 & * \\
\hline
\end{tabular}

Tableau III. - Contrainte et déplacement moyens obtenus pour la charge maximale; calcul avec déplacement imposé. Tableau III. - The mean values of stress and displacement obtained at the maximum load; imposed displacement computation. 
la grave, a permis d'obtenir une répartition de contraintes compatible avec le comportement du matériau. Le modèle retenu est plus réaliste que celui de l'élasticité linéaire, qui est considéré en dimensionnement des chaussées et tient compte de contraintes de traction fictives.

Une étude comparative montre que le chargement avec déplacement imposé est plus « dur » que celui réalisé avec contrainte imposée. Il entraîne des contraintes de cisaillement élevées, et conduit à une charge maximale plus petite que celle obtenue avec contrainte imposée.

Remerciements. L'auteur tient à remercier M. S. Andrieux pour ses conseils, et ses suggestions pour la publication de cet article.

\section{BIBLIOGRAPHIE}

1. AUBRY D., DES CROIX P. (1979), « Numerical algorithm for an elastoplastic constitutive equation with two yield surfaces». $3^{\text {rd }}$ Inter. Conf. on Numerical Methods in Geomechanics, Aachen, vol. 1, pp. 283-288.

2. BARBAS A., FRANK R. (1982), «Utilisation de la méthode des éléments finis en mécanique des sols dans le domaine de l'élastoplasticité». Rapport de recherche, 116, L.C.P.C., Paris.

3. BOUASSIDA M. (1985), "Sur un modèle élastoplastique avec écrouissage pour le calcul des chaussées souples». Thèse de $3^{e}$ cycle, E.N.I.T.

4. BOUASSIDA M., VUEZ A. (1984), "An elastoplastic model for the design of flexible pavements». Proc. VII Congresso Nazionale, AIMETA. Trieste 2-5 ottobre, V-13, pp. 133-144.

5. BURLAND J. (1967), «Deformation of soft clay». Ph. D. Thesis, Cambridge.

6. COULOMB C.A. (1773), "Sur une application des règles de maximis et minimis à quelques problèmes de statique relatifs à l'architecture ». Acad. Sc. Mem. Math. et Phys., 7, pp. 343-382.

7. DHATT G., TOUZOT H. (1981), "Une présentation de la méthode des éléments finis». Maloine S.A., édit. Paris, les presses de l'université LavalQuébec.

8. DRUCKER D.C., PRAGER W. (1951), "Soil Mechanics and plastic analysis of limit design ». Quarterly of Applied Mathematics, vol. 10, pp. 157-175.

9. DRUCKER D.C., GIBSON R.E., HENKEL D.J. (1957), «Soil Mechanics and workhardening theories of plasticity». A.S.C.E., vol. 122, pp. 338-346.

10. FRÉMOND M. (1982), "Méthodes variationnelles en calcul des structures". Cours de D.E.A., E.N.P.C. de Paris.

11. FRIÂA A., MARCHAND J.P., BOUASSIDA M. (1983), «Un modèle de chaussée souple. Application de la méthode des éléments finis ». Annales des Ponts et Chaussées, $2^{e}$ trimestre $n^{\circ} 26$, pp. 23-31.
12. HABIB P., LUONG M.P. (1978), « Sols pulvérulents sous chargements cycliques». Séminaire « Matériaux et structures sous chargements cycliques». École Polytechnique, Paris, 28-29 septembre, pp. 39-63.

13. KALTEZIOTIS N.A., MENZIES B.K., TANZI A.I. (1984), "A bearing capacity correction for strainsoftening saturated clays». Ground Engineering, pp. 30-31.

14. KIRKPATRICK W.M. (1961), «Discussion of soil properties and their measurements ». Proc. of the $5^{\text {th }}$ Int. Conf., Soil Mech. Found. Eng., Paris, pp. 131-133.

15. LADE P.V., DUNCAN J.M. (1975), “Elastoplastic stress strain theory for cohesionless soil ». Journal of the Geotechnical Engineering Division. Proc. of the A.S.C.E., 102, GT 1, pp. 51-68.

16. LADE P.V. (1977), «Elastoplastic stress-strain theory for cohesionless soil with curved yield surfaces». Int. Jour. Solids Structures, vol. 13, $\mathrm{n}^{\circ} 11$, pp. 1019-1035.

17. LORET B. (1981), "Formulation d'une loi de comportement élastoplastique des matériaux granulaires». Thèse Dr. Ing., E.N.P.C. Paris.

18. LUONG M.P. (1978), "État caractéristique du sols. C.R. Acad. Sc., Paris, t. 287, B, pp. 305307.

19. MANDEL J. (1978), «Propriétés mécaniques des matériaux». Edition Eyrolles, Paris.

20. MARTINEZ J. (1980), "Contribution au dimensionnement des chaussées souples; comportement des matériaux et méthode de calcul ». Thèse Dr. Ing., I.N.S.A. de Rennes.

21. MROZ Z., NORRIS V.A. (1982), "Elastoplastic and viscoplastic constitutive models for soils with application to cyclic loading». Soil Mechanics, transient and cyclic loads, edit. G.N. Pand and O.C. Zienkiewicz; chap. 8, pp. 173-217.

22. NGUYEN Q.S., BUI H.D. (1974), «Sur les matériaux élastoplastiques à écrouissage positif ou négatif ». J.M.T.A., vol. 13, $n^{\circ} 2$, pp. 321-342.

23. PENDER M.Z. (1977), «A unified model for soil stress-strain behaviour ». I.C.S.M.F.E., Tokyo.

24. PRÉVOST J.H., HOËG K. (1975), «Effective stress-strain strenthg model for soils ». Journal of the Geotechnical Engineering Division of the A.S.C.E., 101, GT 3.

25. PRÉVOST J.H. (1977), « Mathematical modeling of monotonic and cyclic undrained clay behaviour». Int. Jour. of Num. and Anal. Meth. in Geom., vol. 1, n०2, pp. 159-216.

26. PRÉVOST J.H. (1979), « Mathematical modeling of soil stress-strain strength behaviour». $3^{\text {rd }}$ Int. Conf. on Num. Meth. in Geom., Aachen, vol. 1, pp. 347-361.

27. ROSCOE K.H., SCHOFIELD A.N., WROTH C.P. (1958), "On the yielding of soils *. Géotechnique, 9 , pp. 71-83. 
28. ROSCOE K.H., SCHOFIELD A.N., THURAIRAJAH (1963), "Yielding of clays in states wetter than critical». Géotechnique, vol. 13, $n^{\circ} 3$, pp. 211-240.

29. ROSCOE K.H., BURLAND J.B. (1968), «On the generalized stress-strain behaviour of wet clay. engineering plasticity». Cambridge-HeymanLeckye editors.

30. TANZI A.I., KALTEZIOTIS N.A., MENZIES B.K. (1982), «Finite element analysis of strip footing on strain-softening clay». Proc. Int. Symp. on Numerical Models in Geomechanics. Balkema, Rotterdam.

31. VERMEER P.A. (1978), «A double hardening model for sand». Géotechnique, 28, 4, pp. 413-433.
32. VERMEER P.A., DE BROST R. (1984), «Non associated plasticity for soils, concrete and rocks». Journal Heron, vol. 29, n³, pp. 1-64.

33. WILDE P. (1977), "Simple model of granular material in finite element solutions». Arch. Hydrot., 24, 21, pp. 175-187.

34. WILDE P. (1979), «Mathematical and physical foundations of elastoplastic models for granular media». Colloque Franco-Polonais, Paris 25 30 mars (traduction française), L.C.P.C., pp. 5 27.

35. WROTH P., SCHOFIELD A. (1968), «Critical state soils mechanics ». Mac Graw Hill Editor. 